\title{
Sodium butyrate inhibits high cholesterol-induced neuronal amyloidogenesis by modulating NRF2 stabilization-mediated ROS levels: involvement of NOX2 and SOD1
}

Seo Yihl Kim', Chang Woo Chae', Hyun Jik Lee ${ }^{2,3}$, Young Hyun Jung ${ }^{1}$, Gee Euhn Choi', Jun Sung Kim @', Jae Ryong Lim', Joo Eun Lee ${ }^{1}$, Ji Hyeon Cho ${ }^{1}$, Hansoo Park ${ }^{4,5}$, Changho Park ${ }^{5}$ and Ho Jae Han ${ }^{1}$

\begin{abstract}
The gut-brain axis is currently being studied as a therapeutic strategy for neurological diseases, especially Alzheimer's disease (AD). Obesity results in the gut microbiota dysbiosis, which includes butyrate-producing bacteria are reduced. Although sodium butyrate $(\mathrm{NaB})$ has emerged as the potential therapeutic substance in $\mathrm{AD}$, there is a lack of detailed results into what signaling pathways affect amyloidogenesis in AD induced by obesity. Thus, we investigated the regulatory role of $\mathrm{NaB}$ on amyloidogenesis in neuronal cells under high cholesterol. In our results, we verified that increased amyloid $\beta$ peptide (A $\beta$ ) accumulation in the brain of obese mice and a reduction in butyrate-producing bacteria due to the gut microbiota dysbiosis induced by obesity. We showed that $\mathrm{NaB}$ decreased the expression levels of beta-site amyloid precursor protein cleaving enzyme 1 (BACE1) and A $\beta$ accumulation induced by high cholesterol in SK-N-MC cells. We demonstrated that NaB was absorbed in cells through sodium-coupled monocarboxylate transporter 1 (SMCT1) and then inhibited high cholesterol-induced A $\beta$ accumulation. Subsequently, we also observed that reactive oxygen species (ROS) were overproduced because of increased NADPH oxidase 2 (NOX2) expression under high cholesterol. Meanwhile, NaB decreased NOX2 levels through a reduction of NF-kB activity, which ultimately inhibited A $\beta$ accumulation caused by high cholesterol. We demonstrated that NaB increased the expression levels of p21 under high cholesterol, contributing to p21/NRF2 (Nuclear factor erythroid 2-related factor 2) colocalization, which leads to NRF2 stabilization. NRF2 stabilization causes NF-KB inactivation, followed by NOX2 suppression and superoxide dismutase 1 (SOD1) upregulation. Thus, $\mathrm{NaB}$ with SOD1 silencing under high cholesterol did not eliminate excessive ROS, and eventually resulted in $A \beta$ accumulation. In conclusion, we demonstrated that $\mathrm{NaB}$ prevents excessive ROS through NOX2 suppression and SOD1 upregulation by p21/NRF2 pathway, which is critical for inhibiting BACE1-dependent amyloidogenesis in neuronal cells exposed to high cholesterol environment.
\end{abstract}

\footnotetext{
Correspondence: Ho Jae Han (hjhan@snu.ac.kr)

'Department of Veterinary Physiology, College of Veterinary Medicine,

Research Institute for Veterinary Science, and BK21 PLUS Program for Creative Veterinary Science Research, Seoul National University, Seoul 08826, Republic of Korea

${ }^{2}$ Laboratory of Veterinary Physiology, College of Veterinary Medicine,

Chungbuk National University, Cheongju, Chungbuk 28644, South Korea

Full list of author information is available at the end of the article

Edited by M. Agostini
}

\section{Introduction}

The influence of gut microbiota on central nervous system (CNS) function is called the "gut-brain axis"1. The gut-brain axis is emerging as a therapeutic strategy for neurological diseases, especially $\mathrm{AD}$; thus, many studies are under way focusing on the alteration of gut microbiome and metabolites of gut microbiota to prevent $\mathrm{AD}^{2-4}$. Accumulating studies have shown that the gut microbiota

\section{(c) The Author(s) 2020}

(c) (i) Open Access This article is licensed under a Creative Commons Attribution 4.0 International License, which permits use, sharing, adaptation, distribution and reproduction cc) in any medium or format, as long as you give appropriate credit to the original author(s) and the source, provide a link to the Creative Commons license, and indicate if changes were made. The images or other third party material in this article are included in the article's Creative Commons license, unless indicated otherwise in a credit line to the material. If material is not included in the article's Creative Commons license and your intended use is not permitted by statutory regulation or exceeds the permitted use, you will need to obtain permission directly from the copyright holder. To view a copy of this license, visit http://creativecommons.org/licenses/by/4.0/. 


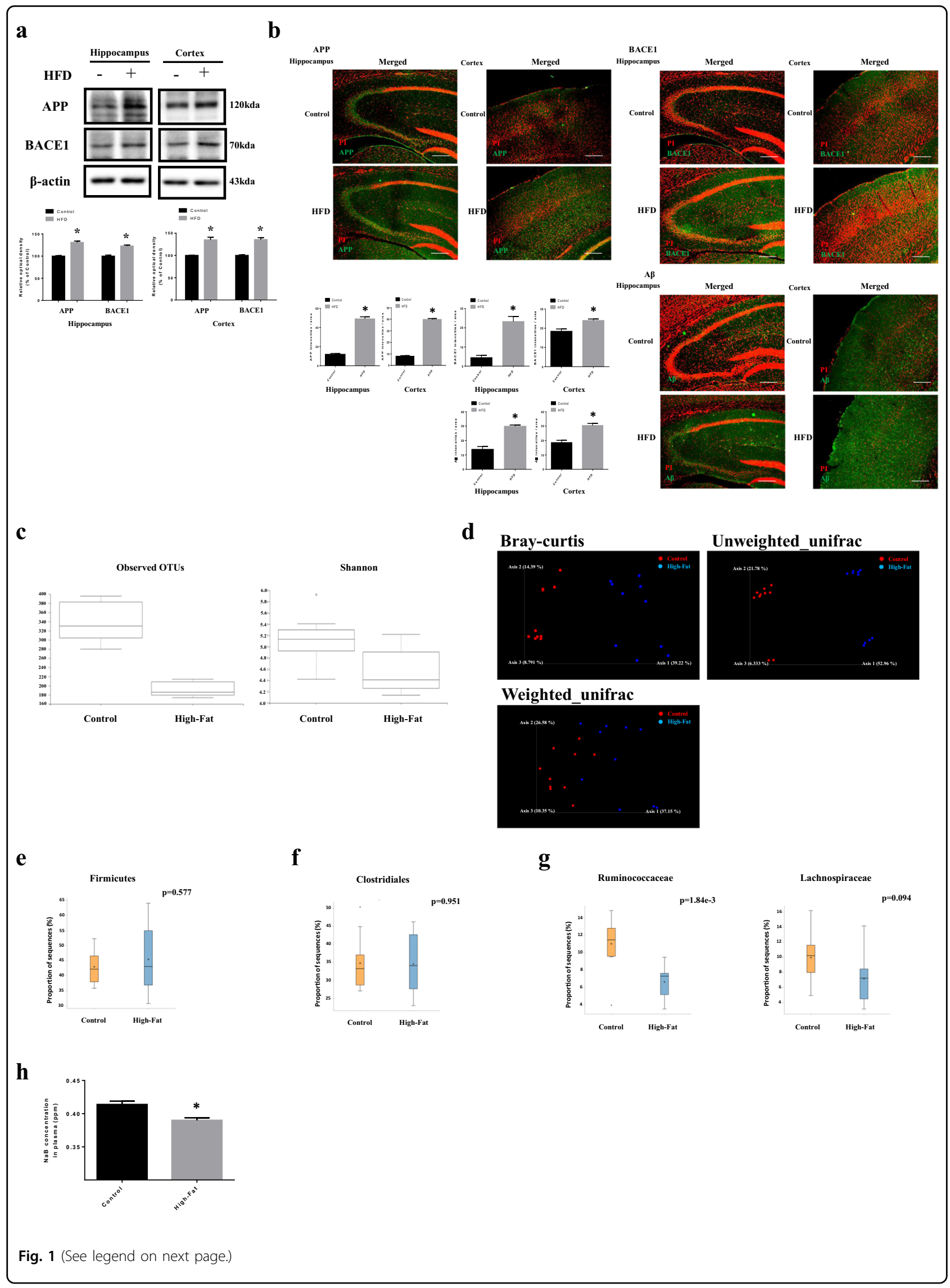


(see figure on previous page)

Fig. 1 BACE1 expression and $A \beta$ accumulation in the brain of the obesity model and the alteration of the gut microbiota composition in the obesity model. a Brains of 20 weeks old ICR mice, which were fed with HFD for 14 weeks were extracted. The extracted brains were cryosectioned coronally, and separated into hippocampus and cortex. The expression levels of APP, BACE1, and $\beta$-actin were analyzed by western blot. $\beta$-actin was used as a loading control. Data are presented as a mean \pm S.E.M. $n=4$. $\mathbf{b}$ Brain samples for immunohistochemistry were immunostained with APP, BACE1, and AB and PI. Images shown in result are representative. All scale bars, $25 \mu \mathrm{m}$ (magnification, $\times 100$ ) $\mathbf{c}$ Alpha diversity between control and high-fat; left panel: Observed OTUs, right panel: Shannon's diversity index. d Beta diversity between control (•) and high-fat (•); Bray-Curtis, unweighted UniFrac distance, weighted UniFrac distance. e- $\mathbf{g}$ STAMP analysis; e Differences in relative abundance of firmicutes between groups at the phylum level, $\mathbf{f}$ Differences in relative abundance of clostridiales between groups at the order level, $\mathbf{g}$ Differences in relative abundance of ruminococcaceae and lachnospiraceae between groups at the family level. $\mathbf{h}$ The concentration of NaB in plasma of mice was measured by GC-MS. $\mathrm{NaB}$ concentration data are presented as a mean \pm S.E.M. $n=4 .{ }^{*} p<0.05$ versus control. All blot and immunofluorescence images shown are representative.

composition in obese patients showed that butyrateproducing bacteria are reduced in the gut ${ }^{5,6}$. In previous studies, $\mathrm{NaB}$ was already proposed as a therapeutic substance for $\mathrm{AD}$ through antioxidant and anti-inflammatory functions ${ }^{7,8}$. However, there are few studies on how the mechanism of $\mathrm{NaB}$ regulates obesity-induced $\mathrm{AD}$. Therefore, the study of how $\mathrm{NaB}$ affects amyloidogenesis caused by obesity is meaningful. $\mathrm{NaB}$ plays diverse biological roles, and it affects cell signaling pathways through mitochondrial activity regulation, histone deacetylase (HDAC) inhibitor, and G-protein coupled receptor $(\text { GPCR })^{9-11}$. However, the specific mechanisms by which $\mathrm{NaB}$ inhibits $\mathrm{A} \beta$ secretion in neurons remain still unclear. Therefore, it will be a key factor of the mechanism whether $\mathrm{NaB}$ is absorbed into neurons and acts as an HDAC inhibitor or affects cell signaling pathways as a GPCR ligand.

Many studies have shown various evidence that obesity induces $\mathrm{AD}$, and obesity has been also reported as a risk factor that induces AD independently of diabetes mellitus $^{12-14}$. One of typical characteristics found in obese patients is high-cholesterol levels, which is also a major cause of the pathogenesis of $\mathrm{AD}^{12,15,16}$. When cells are exposed to high-cholesterol environment, they produce excessive $\operatorname{ROS}^{17}$. Neurons under high cholesterol also produce excessive ROS, which is known as one of the major contributors to amyloidogenesis ${ }^{18}$. There are generally two types of ROS sources in the cells; one is NOX and the other is mitochondria ${ }^{19}$. Previous studies reported that the critical factor of ROS in the CNS is $\mathrm{NOX}^{20}$, but further studies are needed to determine the specific source of excessive ROS produced in neurons under high cholesterol. Thus, it is essential to investigate the major source of excessive ROS caused by high cholesterol and how $\mathrm{NaB}$ affects cell signaling pathways associated with NOX or mitochondrial ROS (mtROS).

The most prominent transcription factor that regulates expression of antioxidant enzymes is an $\mathrm{NRF}^{21}$. In response to oxidative damages, cells are protected by NRF2/Keap1/ARE antioxidant pathway ${ }^{21,22}$. However, a study reported that overproduced ROS in response to oxidative damages make antioxidant defense vulnerable $\mathrm{e}^{23}$. Therefore, since $\mathrm{NaB}$ has been shown to act as an activator of $\mathrm{NRF}^{24}$, the mechanism by which $\mathrm{NaB}$ activates NRF2 under high cholesterol will be meaningful. Previous studies reported that $\mathrm{NaB}$ increases p21 levels, which contributes to NRF2 stabilization by binding to NRF2 ${ }^{25,26}$. Therefore, it seems necessary to investigate the specific mechanism by which $\mathrm{NaB}$ stabilizes NRF2 through p21/ NRF2 pathway.

In the present study, we prepared a high fat diet (HFD)induced mice models of obesity for AD because HFD feeding has been reported to cause cognitive impairment ${ }^{27-29}$. To investigate the exact mechanisms of the inhibitory effect of $\mathrm{NaB}$ on amyloidogenesis, we used SKN-MC cells, which are a human neuronal epithelioma cell line ${ }^{30}$. SK-N-MC cells are well-stabilized cell line and widely used as a model for studying cellular signal transduction in many $\mathrm{AD}$ studies ${ }^{31,32}$. To address this issue, we identified the mechanisms of how $\mathrm{NaB}$ reduces high cholesterol-induced ROS and investigated the protective role of $\mathrm{NaB}$ against amyloidogenesis caused by high cholesterol.

\section{Result \\ BACE1 expression and $A \beta$ accumulation in the brain of the obesity model and the alteration of the gut microbiota composition in the obesity model}

We verified BACE1 expression and $A \beta$ accumulation in the hippocampus and cortex to confirm the effect of obesity. We found that amyloid precursor protein (APP) and BACE1 levels were higher in HFD-fed mice than in normal diet (ND)-fed mice (Fig. 1a). In immunohistochemistry results, APP, BACE1, and $\mathrm{A} \beta$ were also increased in the hippocampus and cortex in brains of HFD-fed mice (Fig. 1b). The analysis of gut microbiota diversity mainly includes alpha diversity and beta diversity. In brief, alpha diversity identifies the distribution of various microorganisms in a sample and beta diversity is a method of checking whether the sample diversity is similar $^{33,34}$. The analyses showed that the gut microbiota diversity was lower in HFD-fed mice than in ND-fed mice 
a

c
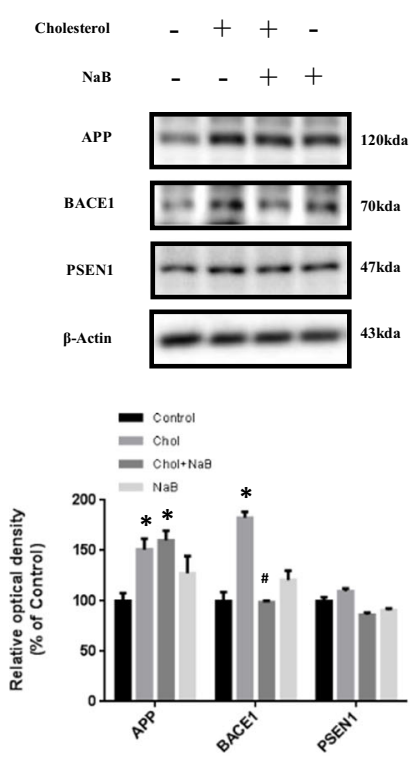

e

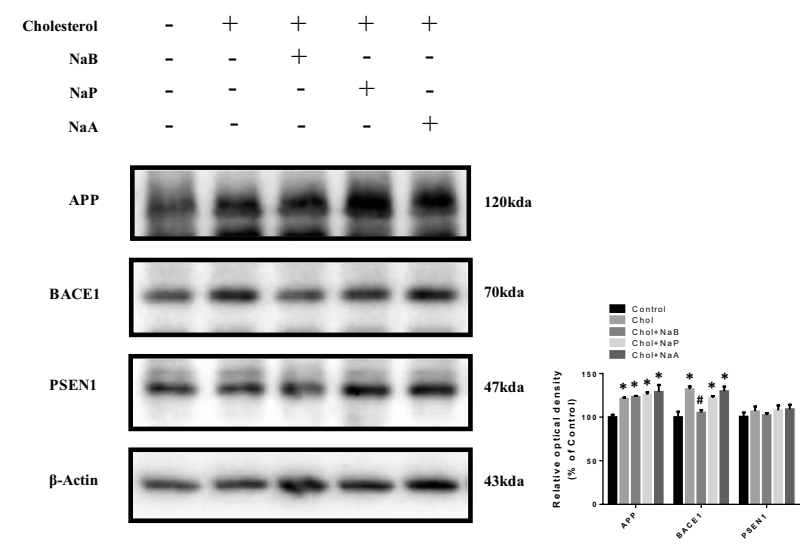

b

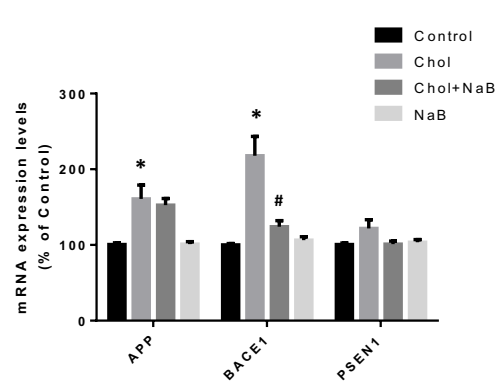

d
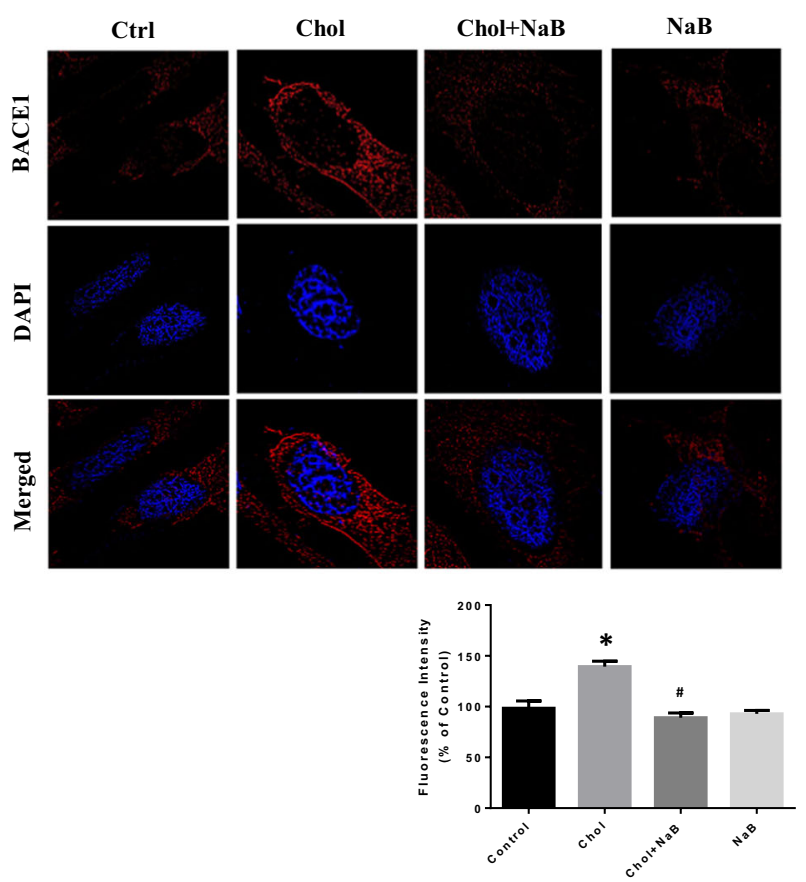

f

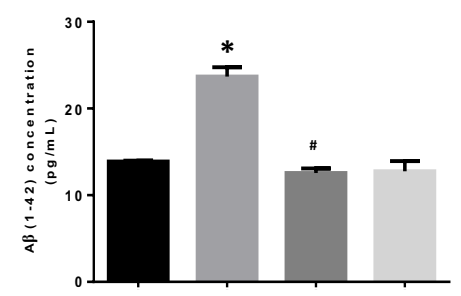

Cholesterol

$\mathrm{NaB}$

Fig. 2 (See legend on next page.) 
(see figure on previous page)

Fig. 2 Effect of $\mathrm{NaB}$ on high-cholesterol-induced BACE1 expression and $\mathrm{A} \boldsymbol{\beta}$ accumulation. a SK-N-MC cells were treated with high cholesterol $(25 \mu \mathrm{M})$ for various time (0-48 h). APP and BACE1 were analyzed by western blot. $\beta$-actin was used as a loading control. $n=4$. b Cells were pretreated with $\mathrm{NaB}(500 \mu \mathrm{M})$ for $30 \mathrm{~min}$ prior to treatment of high cholesterol for $24 \mathrm{~h}$. The mRNA expression levels of APP, BACE1, and PSEN1 were analyzed by quantitative real-time PCR. Data were normalized by the ACTB mRNA expression levels. $n=4$. c Cells were pretreated with $\mathrm{NaB}$ for 30 min prior to treatment of high cholesterol for $24 \mathrm{~h}$. The expression levels of APP, BACE1, and PSEN1 were analyzed by western blot. $\beta$-actin was used as a loading control. $n=4$. $\mathbf{d}$ Cells were pretreated with NaB for 30 min prior to treatment of high cholesterol for $24 \mathrm{~h}$ and immunostained with BACE1 antibody. Scale bars are $8 \mu \mathrm{m}$ (magnification, $\times 1,000) . n=3$. e Cells were pretreated with $\mathrm{NaB}, \mathrm{NaP}(500 \mu \mathrm{M})$, and NaA $(500 \mu \mathrm{M})$ for 30 min prior to treatment of high cholesterol for $24 \mathrm{~h}$. The expression levels of APP, BACE1, and PSEN1 were analyzed by western blot. $\beta$-actin was used as a loading control. $n=3$. f Cells were pretreated with $\mathrm{NaB}$ for 30 min prior to treatment for high cholesterol for $72 \mathrm{~h}$. A $\beta$ concentration of medium samples was detected by using ELISA kit. Data are presented as a mean \pm S.E.M. $n=4 .{ }^{*} p<0.05$ versus control, ${ }^{*} p<0.05$ versus high-cholesterol treatment. All blot and immunofluorescence images shown are representative.

(Fig. 1c, d). Firmicutes to which most butyrate-producing bacteria belong in phylum level were not altered in the HFD group compared to the ND group (Fig. 1e). Clostridiales to which most butyrate-producing bacteria belong in order level were also not changed in the HFD group (Fig. 1f). In the case of Ruminococcaceae and Lachnospiraceae to which butyrate-producing bacteria belong in family level ${ }^{35}$, there was a significant reduction in HFD-fed mice (Fig. 1g). We presented the list of $p$ value in phylum, order, and family level of gut microbiota in the obesity model in Supplementary Table S1. Furthermore, we measured concentration of $\mathrm{NaB}$ in plasma from mice by using gas chromatography-mass spectrometry (GC-MS). The concentration of $\mathrm{NaB}$ in plasma from HFD-fed mice was lower than ND-fed mice (Fig. 1h). Subsequently, we also measured high cholesterol levels in plasma from mice. Our data showed that total cholesterol levels were significantly higher in HFD-fed mice [Supplementary Fig. S1].

\section{Effect of $\mathrm{NaB}$ on high cholesterol-induced BACE1 expression and $A \beta$ accumulation}

To confirm effect of high cholesterol on $A \beta$, we analyzed APP and BACE1 levels in SK-N-MC cells treated with high cholesterol for 0 to $48 \mathrm{~h}$. High cholesterol increased APP and BACE1 levels at 24-48 h (Fig. 2a). We investigated whether $\mathrm{NaB}$ affects APP, BACE1, and presenilin-1 (PSEN1) levels. Our data showed that $\mathrm{NaB}$ decreased only high cholesterol-induced BACE1 (Fig. 2b, c). In immunofluorescence staining results, the fluorescence intensities of BACE1 under high cholesterol were increased, but recovered by $\mathrm{NaB}$ (Fig. 2d). These results indicated that $A \beta$ secretion induced by high cholesterol was dependent on BACE1, and $\mathrm{NaB}$ decreased BACE1 levels, which eventually blocked $A \beta$ accumulation. We also performed $B A C E 1$ siRNA transfection to verify that $A \beta$ secretion caused by high cholesterol depends on BACE1. Our data showed that $A \beta$ levels were decreased by $B A C E 1$ siRNA transfection under high cholesterol [Supplementary Fig. S2]. Next, we compared effect of short chain fatty acids (SCFAs) on APP, BACE1, and
PSEN1 levels. Sodium propionate $(\mathrm{NaP})$ and sodium acetate $(\mathrm{NaA})$ did not significantly affect anything, but $\mathrm{NaB}$ influenced only BACE1 levels (Fig. 2e). In addition, when $A \beta$ levels were measured by enzyme-linked immunosorbent assay (ELISA), the levels treated with $\mathrm{NaB}$ under high cholesterol were decreased (Fig. 2f).

\section{Involvement of SMCT1 in inhibitory effect of $\mathrm{NaB}$ on high cholesterol-induced ROS generation, BACE1 expression, and $A \beta$ accumulation}

We investigated whether the reason for a reduction of high cholesterol-induced $\mathrm{ROS}$ by $\mathrm{NaB}$ is through acting as an HDAC inhibitor or as a GPCR ligand. In our data, high cholesterol-induced ROS were reduced by $\mathrm{NaB}$, but when both $\mathrm{NaB}$ and ibuprofen (SMCT inhibitor) were pretreated under high cholesterol, ROS were not decreased (Fig. 3a). However, when pertussis toxin (PTX, Goi inhibitor) was pretreated instead of ibuprofen, ROS caused by high cholesterol were decreased (Fig. 3b). Consistent with Fig. 3a, b, Our data showed that excessive ROS were not reduced by $\mathrm{NaB}$ and ibuprofen, but by $\mathrm{NaB}$ and PTX by using flow cytometry (Fig. 3c, d). However, there is a study that ibuprofen has an antioxidant effect ${ }^{36}$. Thus, we performed SMCT1 siRNA transfection: the ratio of SMCT1 is high in neurons ${ }^{37}$. In our results, high-cholesterolinduced ROS were reduced by NT siRNA transfection and $\mathrm{NaB}$, but SMCT1 siRNA transfection and $\mathrm{NaB}$ led to ROS accumulation (Fig. 3e). Furthermore, BACE1 levels were decreased by $\mathrm{NaB}$, and increased when both $\mathrm{NaB}$ and ibuprofen were pretreated under high cholesterol (Fig. 3f). In contrast, when PTX was pretreated with $\mathrm{NaB}$, BACE1 levels were decreased under high cholesterol (Fig. $3 g$ ). In addition, BACE1 and $A \beta$ levels were not decreased by SMCT1 siRNA transfection and $\mathrm{NaB}$ under high cholesterol (Fig. 3h, i).

\section{Effect of NaB on high cholesterol-induced NOX2 expression}

To determine main source of ROS produced by high cholesterol, we pretreated Vas2870 (NOX inhibitor) and mitotempo (mtROS inhibitor). High cholesterol-induced 


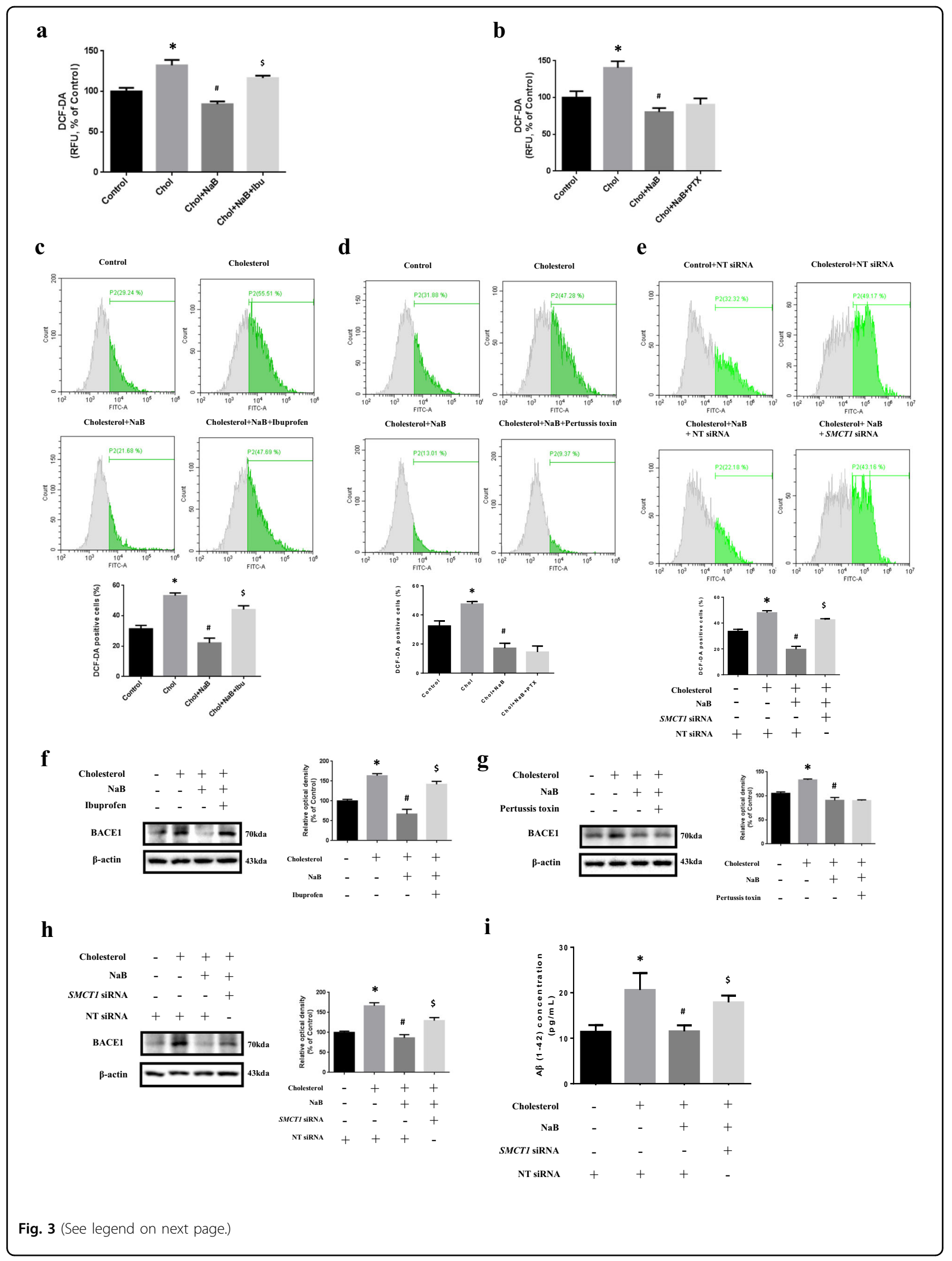


(see figure on previous page)

Fig. 3 Involvement of SMCT1 in inhibitory effect of $\mathrm{NaB}$ on high cholesterol-induced ROS generation, BACE1 expression, and A $\beta$

accumulation. a SK-N-MC cells were pretreated with $\mathrm{NaB}$ and ibuprofen $(500 \mu \mathrm{M})$ for $30 \mathrm{~min}$ prior to treatment of high cholesterol for $48 \mathrm{~h}$ where DCF-DA was detected by luminometer. $n=4 .{ }^{*} p<0.05$ versus control, ${ }^{\#} p<0.05$ versus high cholesterol treatment, ${ }^{\$} p<0.05$ versus treatment of high cholesterol and NaB. b Cells were pretreated with $\mathrm{NaB}$ and PTX (200 nM) for 30 min prior to treatment of high cholesterol for $48 \mathrm{~h}$ where DCF-DA was detected by luminometer. ${ }^{*} p<0.05$ versus control, ${ }^{*} p<0.05$ versus high cholesterol treatment. c Cells were pretreated with NaB and ibuprofen for $30 \mathrm{~min}$ prior to treatment of high cholesterol for $72 \mathrm{~h}$ where ROS with DCF-DA were measured by flowcytometer. Total cell counts $=1.0 \times 10^{4}$ cells. $n=4$. ${ }^{*} p<0.05$ versus control, ${ }^{\#} p<0.05$ versus high cholesterol treatment, ${ }^{\$} p<0.05$ versus treatment of high cholesterol and NaB. $\mathbf{d}$ Cells were pretreated with $\mathrm{NaB}$ and PTX for 30 min prior to treatment of high cholesterol for $72 \mathrm{~h}$ where ROS with DCF-DA were measured by flowcytometer. Total cell counts $=1.0 \times 10^{4}$ cells. $n=4 .{ }^{*} p<0.05$ versus control, ${ }^{\#} p<0.05$ versus high cholesterol treatment. e Cells were transfected with SMCT1 siRNA or NT siRNA for $12 \mathrm{~h}$, and pretreated with $\mathrm{NaB}$ for $30 \mathrm{~min}$ prior to treatment of high cholesterol for $72 \mathrm{~h}$ where ROS with DCF-DA were measured by flowcytometer. Total cell counts $=1.0 \times 10^{4}$ cells. Data are presented as a mean \pm S.E.M. $n=3 .{ }^{*} p<0.05$ versus control with NT siRNA transfection, ${ }^{\#} p<0.05$ versus high cholesterol treatment with NT siRNA transfection, ${ }^{\$} p<0.05$ versus treatment of high cholesterol and NaB with NT siRNA transfection. $\mathbf{f}$ Cells were pretreated with $\mathrm{NaB}$ and ibuprofen for $30 \mathrm{~min}$ prior to treatment of high cholesterol for $24 \mathrm{~h}$. The expression levels of BACE1 were analyzed by western blot. $\beta$-actin was used as a loading control. $n=3 .{ }^{*} p<0.05$ versus control, ${ }^{*} p<0.05$ versus high cholesterol treatment, $\$ p<0.05$ versus treatment of high cholesterol and NaB. $\mathbf{g}$ Cells were pretreated with NaB and PTX for 30 min prior to treatment of high cholesterol for $24 \mathrm{~h}$. The expression levels of BACE1 were analyzed by western blot. $\beta$-actin was used as a loading control. $n=3 .{ }^{*} p<0.05$ versus control, ${ }^{\#} p<0.05$ versus high-cholesterol treatment. $\mathbf{h}$ Cells were transfected with SMCT1 siRNA or NT siRNA for $12 \mathrm{~h}$, and pretreated with NaB for $30 \mathrm{~min}$ prior to treatment of high cholesterol for $24 \mathrm{~h}$. The expression levels of BACE1 were analyzed by western blot. $\beta$-actin was used as a loading control. $n=3 .{ }^{*} p<0.05$ versus control with NT siRNA transfection, ${ }^{\#} p<0.05$ versus high cholesterol treatment with NT siRNA transfection, ${ }^{\$} p<0.05$ versus treatment of high cholesterol and $\mathrm{NaB}$ with NT siRNA transfection. i Cells were transfected with SMCT1 siRNA or NT siRNA for $12 \mathrm{~h}$, and pretreated with $\mathrm{NaB}$ for 30 min prior to treatment of high cholesterol for $72 \mathrm{~h}$. A $\beta$ concentration of medium samples was detected by using ELISA kit. Data are presented as a mean \pm S.E.M. $n=4 .{ }^{*} p<0.05$ versus control with NT siRNA transfection, ${ }^{\#} p<0.05$ versus high cholesterol treatment with NT siRNA transfection, ${ }^{\$} p<0.05$ versus treatment of high cholesterol and NaB with NT siRNA transfection. All blot images shown are representative.

ROS were blocked by Vas2870 but not by mitotempo (Fig. 4a). We also checked mtROS by staining with MitoSOX ${ }^{\mathrm{TM}}$ Red. In our results, mtROS did not show a significant increase (Fig. 4b). Previous studies reported that the most prominent transcription factor of NOX2 is nuclear factor-kappa $B(N F-\kappa B)^{38}$. Our results showed that NF- $\mathrm{kB}$ levels in the nucleus were increased by high cholesterol, but decreased by $\mathrm{NaB}$ (Fig. 4c, d). Previous studies reported that NOX1-4 are major isotypes of NOX expressed in the brain, and there seems to be difference in expression depending on cell types ${ }^{39,40}$. Therefore, we made primers referring to previous studies and gradient PCR was performed with complementary DNA of SK-NMC cells ${ }^{41,42}$. Our results showed that NOX1 and NOX3 were hardly expressed in the cells [Supplementary Fig. S3], so real-time PCR was performed with $N O X 2$ and NOX4. In our results, mRNA expression levels of NOX2 showed greater increase by high cholesterol but decreased by $\mathrm{NaB}$ (Fig. 4e). Consistently, protein levels of NOX2 were increased by high cholesterol but decreased by $\mathrm{NaB}$ (Fig. 4f). We also showed NOX2 levels in the hippocampus and cortex and found that NOX2 levels were higher in HFD-fed mice (Fig. 4g). We further investigated whether NF- $\mathrm{kB}$ is a critical transcription factor for NOX2. The increased NOX2 levels under high cholesterol were decreased by Bay11-7082 (NF-kB inhibitor) (Fig. 4h). Subsequently, increased BACE1 levels and $A \beta$ secretion induced by high cholesterol were decreased by Bay117082 (Fig. 4i, j). We further confirmed the involvement of NF- $\mathrm{B}$ in high cholesterol-induced ROS, NOX2, BACE1, and $A \beta$ through Bay11-7082 and $p 65$ siRNA transfection.
Our data showed that both Bay11-7082 and p65 knockdown decreased ROS, NOX2 and BACE1 levels, and A $\beta$ accumulation caused by high cholesterol [Supplementary Fig. S4]. In our results, increased BACE1 and A $\beta$ levels by high cholesterol were decreased by Vas 2870 and $\mathrm{N}$ acetylcysteine (NAC; ROS scavenger), but not mitotempo (Fig. 4k, l). Finally, to further confirm involvement of NOX2 in high cholesterol-induced BACE1 and A $\beta$, we performed NOX2 siRNA transfection. Our results showed that BACE1 and A $\beta$ levels were decreased by NOX2 siRNA transfection under high cholesterol [Supplementary Fig. S5].

\section{Regulatory role of $\mathrm{NaB}$ in high cholesterol-induced downregulation of p21 and p21/NRF2 colocalization}

We investigated the effect of $\mathrm{NaB}$ on the nuclear translocation of NRF2 under high cholesterol. NRF2 levels in the nucleus were decreased by high cholesterol, but were increased by $\mathrm{NaB}$ (Fig. 5a, b). In our results, activity of specificity protein 1 (Sp1), which is one of the most prominent transcription factors of $\mathrm{p} 21^{43}$, was decreased under high cholesterol, but recovered by $\mathrm{NaB}$ (Fig. 5c, d). Previous study has shown that acetylation of transcription factors affects the nuclear translocation ${ }^{44}$. Therefore, we verified whether the acetylation of $\mathrm{Sp} 1$ facilitates the nuclear translocation of sp1. Pretreatment of ibuprofen and $\mathrm{NaB}$ under high cholesterol resulted in reduction of sp1 nuclear translocation (Fig. 5e). We also confirmed the sp1 activation through acetylation of $\mathrm{Sp} 1$ by pretreating C646 (p300/CBP inhibitor) which inhibits Sp1 acetylation. In our results, nuclear translocation of $\mathrm{Sp} 1$ was decreased 


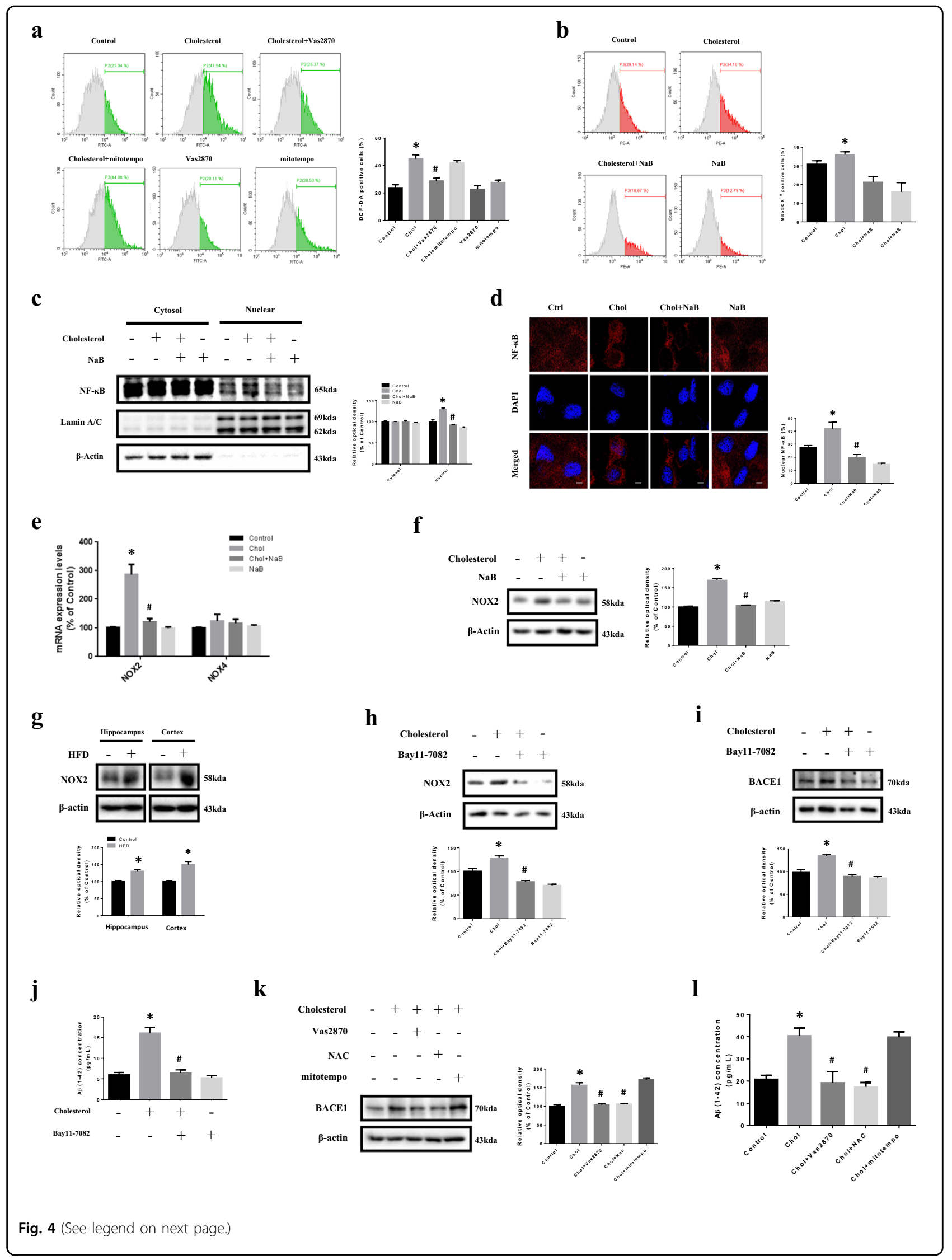


(see figure on previous page)

Fig. 4 Effect of $\mathrm{NaB}$ on high cholesterol-induced NOX2 expression. a SK-N-MC cells were pretreated with Vas $2870(5 \mu \mathrm{M})$ or mitotempo $(2 \mu \mathrm{M})$ for $30 \mathrm{~min}$ prior to high cholesterol treatment for $72 \mathrm{~h}$ where ROS with DCF-DA were measured by flowcytometer. Total cell counts $=1.0 \times 10^{4}$ cells. $n=4 .{ }^{*} p<0.05$ versus control, ${ }^{\#} p<0.05$ versus high cholesterol treatment. b Cells were pretreated with NaB for 30 min prior to high cholesterol treatment for $72 \mathrm{~h}$ where $\mathrm{mtROS}$ with mitoSox ${ }^{\mathrm{TM}}$ red were measured by flowcytometer. Total cell counts $=1.0 \times 10^{4}$ cells. $n=4 .{ }^{\#} p<0.05$ versus high cholesterol treatment. c Cells were pretreated with $\mathrm{NaB}$ for 30 min prior to high cholesterol treatment for $24 \mathrm{~h}$. NF-KB, Lamin A/C, and $\beta$-actin protein levels in cytosolic and nuclear-fractionized samples were analyzed by western blot. $n=3$. ${ }^{*} p<0.05$ versus control, ${ }^{*} p<0.05$ versus high cholesterol treatment. $\mathbf{d}$ Cells were pretreated with $\mathrm{NaB}$ for 30 min prior to high cholesterol treatment for $24 \mathrm{~h}$ and immunostained with NF-kB antibody. Scale bars are $8 \mu \mathrm{m}$ (magnification, $\times 1,000$ ). $n=3 .{ }^{*} p<0.05$ versus control, ${ }^{\#} p<0.05$ versus high cholesterol treatment. e Cells were pretreated with $\mathrm{NaB}$ for $30 \mathrm{~min}$ prior to high cholesterol treatment for $24 \mathrm{~h}$. Data were normalized by the ACTB mRNA expression levels. $n=4$. $\mathbf{f}$ Cells were pretreated with $\mathrm{NaB}$ for $30 \mathrm{~min}$ prior to high cholesterol treatment for $24 \mathrm{~h}$. The expression levels of NOX2 were analyzed by western blot. $\beta$-actin was used as a loading control. $n=4$. ${ }^{*} p<0.05$ versus control, ${ }^{\#} p<0.05$ versus high cholesterol treatment. $\mathbf{g}$ Brains of 20 weeks mice fed with HFD for 14 weeks were extracted. The expression levels of NOX2 were analyzed by western blot. $\beta$-actin was used as a loading control. $n=3 .{ }^{*} p<0.05$ versus control. $\mathbf{h}$,

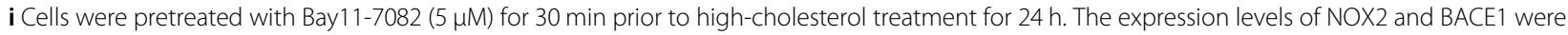
analyzed by western blot. $\beta$-actin was used as a loading control. $n=4$. j Cells were pretreated with Bay 11-7082 for 30 min prior to high cholesterol treatment for $72 \mathrm{~h}$. A $\beta$ concentration of medium samples was detected by using ELISA kit. $n=4$. $\mathbf{k}$ Cells were pretreated with Vas 2870 or NAC ( 5 mM) or mitotempo for $30 \mathrm{~min}$ prior to high-cholesterol treatment for $24 \mathrm{~h}$. The expression levels of BACE1 were analyzed by western blot. $\beta$-actin was used as a loading control. $n=4$. I Cells were pretreated with Vas 2870 or NAC or mitotempo for 30 min prior to high-cholesterol treatment for $72 \mathrm{~h}$. A $\beta$ concentration of medium samples was detected by using ELISA kit. Data are presented as a mean \pm S.E.M. $n=4 .{ }^{*} p<0.05$ versus control, ${ }^{\#} p<0.05$ versus high cholesterol treatment. All blot and immunofluorescence images shown are representative.

after pretreating with $\mathrm{C} 646$ and $\mathrm{NaB}$ under high cholesterol (Fig. 5f), and we finally verified the regulatory role of $\mathrm{NaB}$ on Sp1 acetylation under high cholesterol. Our immunoprecipitation data showed that the acetylation of sp1 reduced by high cholesterol was recovered by $\mathrm{NaB}$ (Fig. 5g). The data showed that $\mathrm{p} 21$ levels were decreased by high cholesterol, but were recovered by $\mathrm{NaB}$ (Fig. 5 h). We further showed p21 levels in the hippocampus and cortex and found that p21 levels were lower in HFD-fed mice (Fig. 5i). Subsequently, we presented that colocalization of p21 and NRF2 was decreased by high cholesterol, but increased by $\mathrm{NaB}$ (Fig. 5j). We further presented results which showed the effect of $\mathrm{NaB}$ via NRF2, Sp1, and p21 on increased ROS, BACE1, and A $\beta$ caused by high cholesterol [Supplementary Fig. S6-8]. There are studies demonstrating the crosstalk between NRF2 and NF$\mathrm{KB}^{45,46}$. Therefore, we confirmed whether the nuclear translocation of NF- $\mathrm{kB}$ was increased by NRF2 siRNA transfection. Our results showed that NF- $\kappa B$ levels in the nucleus were increased by NRF2 siRNA transfection (Fig. $5 \mathrm{k}, \mathrm{l})$ and NOX2 levels were also increased by NRF2 siRNA transfection under high cholesterol (Fig. 5m).

\section{Effect of NaB on high cholesterol-induced ROS generation, BACE1 expression, and $A \beta$ accumulation through SOD1 expression}

We investigated that certain antioxidant enzymes which are products of NRF2 were significantly changed when treated with high cholesterol. Our results showed that only SOD1 levels were decreased by high cholesterol and NRF2 silencing further decreased SOD1 levels under high cholesterol, but the others were not significantly altered (Fig. 6a). We also investigated antioxidant enzymes whose expression is suppressed under high cholesterol and recovered by $\mathrm{NaB}$. Consistent with Fig. 6a, SOD1 levels were decreased by high cholesterol, but recovered by $\mathrm{NaB}$ while the others were not significantly changed (Fig. 6b, c). We also showed SOD1 levels in the hippocampus and cortex and found that SOD1 levels were lower in HFD-fed mice (Fig. 6d). To determine whether $\mathrm{NaB}$ removes excessive ROS through SOD1, ATN-224 (SOD1 inhibitor) was pretreated with $\mathrm{NaB}$ under high cholesterol. Increased ROS, $\mathrm{BACE} 1$, and $\mathrm{A} \beta$ levels by high cholesterol were decreased by $\mathrm{NaB}$, but were not reduced by $\mathrm{NaB}$ and ATN-224 (Fig. 6e-h). To verify more clearly the effect of $\mathrm{NaB}$ on high cholesterol-induced ROS, BACE1, and $\mathrm{A} \beta$ levels through SOD1 expression, we performed SOD1 siRNA transfection. Consistently, our data showed that increased ROS, BACE1, and $A \beta$ levels by high cholesterol were reduced by $\mathrm{NaB}$, but were not recovered by $\mathrm{NaB}$ and SOD1 siRNA transfection [Supplementary Fig. S9].

\section{Discussion}

This study demonstrates the antioxidant effect of $\mathrm{NaB}$ on high cholesterol-induced ROS in SK-N-MC cells, ultimately inhibiting BACE1-dependent amyloidogenesis caused by excessive ROS. Although the antioxidant effect of $\mathrm{NaB}$ is well known ${ }^{8,47}$, inhibitory effect of $\mathrm{NaB}$ on $\mathrm{A} \beta$ secretion through the antioxidant effect in neurons under high cholesterol has not been reported. The reason for targeting $\mathrm{NaB}$ as a potential therapeutic substance in $\mathrm{AD}$ was inspired by "gut-brain axis". We focused on the microbiota composition in obese patients who are more likely to get $\mathrm{AD}$, because butyrate-producing bacteria have been observed to decrease in the gut of obese patients. Therefore, we investigated the butyrateproducing bacteria that generally belong to Firmicutes at phylum level: in previous studies, the alteration of 


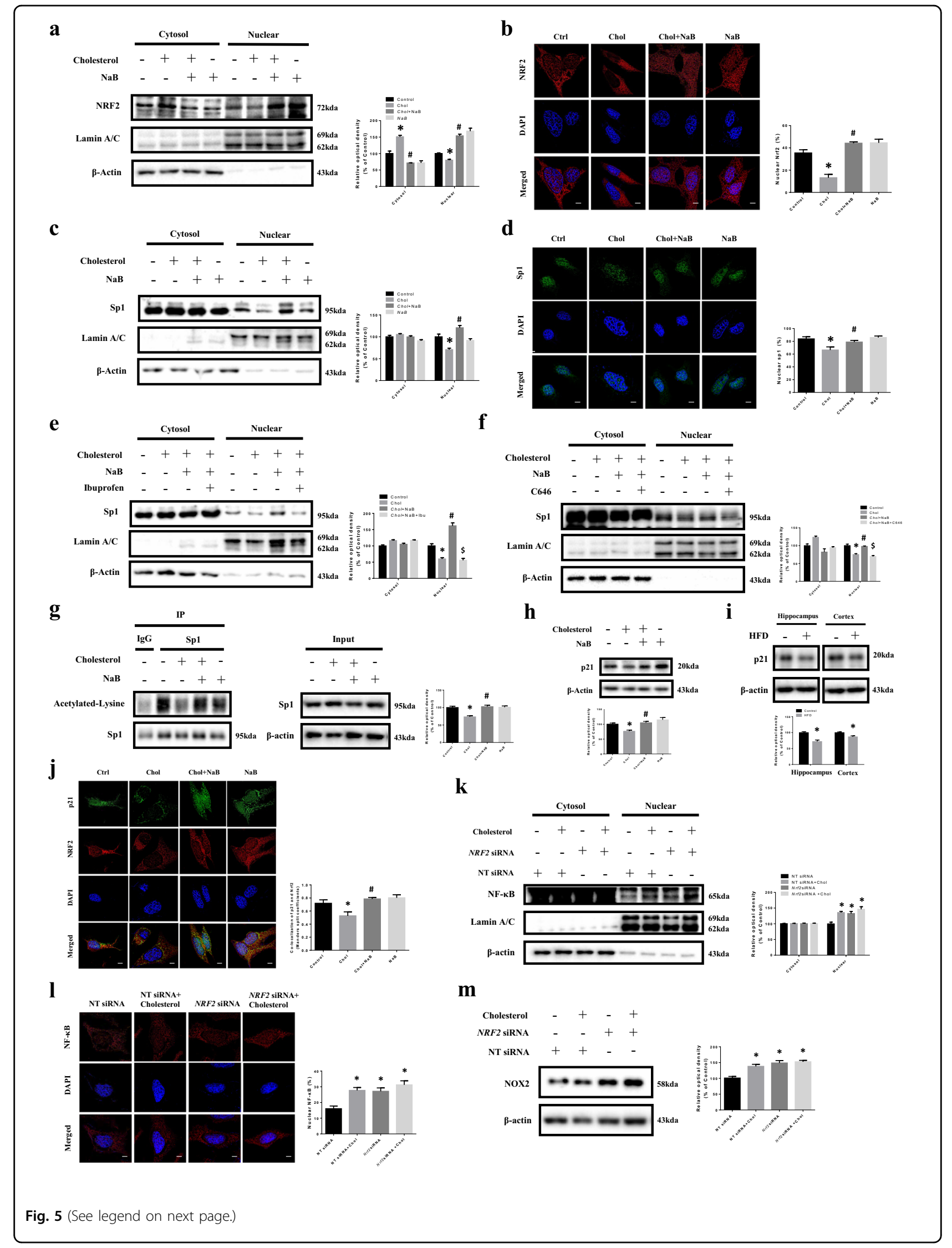


(see figure on previous page)

Fig. 5 Regulatory role of $\mathrm{NaB}$ in high cholesterol-induced downregulation of p21 and p21/NRF2 colocalization. a SK-N-MC cells were pretreated with $\mathrm{NaB}$ for 30 min prior to high cholesterol treatment for $24 \mathrm{~h}$. NRF2, Lamin A/C, and $\beta$-actin protein levels in cytosolic and nuclearfractionized samples were analyzed by western blot. $n=3$. b Cells were immunostained with NRF2 antibody. Scale bars are $8 \mu m$ (magnification, $\times 1,000)$. $n=3$. c SK-N-MC cells were pretreated with NaB for 30 min prior to high cholesterol treatment for $24 \mathrm{~h}$. Sp1, Lamin A/C, and $\beta$-actin protein levels in cytosolic and nuclear-fractionized samples were analyzed by western blot. $n=3$. d Cells were immunostained with sp1 antibody. Scale bars are $8 \mu \mathrm{m}$ (magnification, $\times 1,000$ ). $n=4 .{ }^{*} p<0.05$ versus control, ${ }^{*} p<0.05$ versus high cholesterol treatment. e Cells were pretreated with $\mathrm{NaB}$ and ibuprofen for $30 \mathrm{~min}$ prior to treatment of high cholesterol for $24 \mathrm{~h}$. Sp1, Lamin A/C, and $\beta$-actin protein levels in cytosolic and nuclear-fractionized samples were analyzed by western blot. $n=3$. f Cells were pretreated with $\mathrm{NaB}$ and C646 for 30 min prior to treatment of high cholesterol for $24 \mathrm{~h}$. Sp1, Lamin A/C, and $\beta$-actin protein levels in cytosolic and nuclear-fractionized samples were analyzed by western blot. $n=3 .{ }^{*} p<0.05$ versus control, ${ }^{\#} p<0.05$ versus high cholesterol treatment, ${ }^{\$} p<0.05$ versus treatment of high cholesterol and NaB. $\mathbf{g}$ Cells were pretreated with NaB for $30 \mathrm{~min}$ prior to high cholesterol treatment for $24 \mathrm{~h}$. Co-immunoprecipitation of acetylated-lysine with lgG and Sp1 was shown in left panel. Total protein expressions in lysate were shown in right panel. $n=3$. $\mathbf{h}$ Cells were pretreated with $\mathrm{NaB}$ for 30 min prior to high cholesterol treatment for $24 \mathrm{~h}$. The expression levels of $p 21$ were analyzed by western blot. $\beta$-actin was used as a loading control. ${ }^{*} p<0.05$ versus control, ${ }^{*} p<0.05$ versus high-cholesterol treatment. i Brains of 20 weeks mice fed with HFD for 14 weeks were extracted. The expression levels of p21 were analyzed by western blot. $\beta$-actin was used as a loading control. $n=3 .{ }^{*} p<0.05$ versus control. $\mathbf{j}$ Cells were pretreated with NaB for 30 min prior to high cholesterol treatment for $24 \mathrm{~h}$ and immunostained with p21 and NRF2 antibodies. Colocalization of p21 (green) and NRF2 (red) was visualized with SRRF imaging system. Scale bars are $8 \mu \mathrm{m}$ (magnification, $\times 1,000$ ). $n=4 .{ }^{*} p<0.05$ versus control, ${ }^{\#} p<0.05$ versus high cholesterol treatment. $\mathbf{k}$ Cells were transfected with NRF2 siRNA or NT siRNA for $12 \mathrm{~h}$, and treated with high cholesterol for $24 \mathrm{~h}$. NF-KB, Lamin A/C, and $\beta$-actin protein levels in cytosolic and nuclear-fractionized samples were analyzed by western blot. $n=3$. I Cells were immunostained with NF-kB antibody. Scale bars are $8 \mu \mathrm{m}$ (magnification, $\times 1,000$ ). $n=4$. $\mathbf{m}$ Cells were transfected with NRF2 siRNA or NT siRNA for $12 \mathrm{~h}$, and treated with high cholesterol for $24 \mathrm{~h}$. The expression levels of NOX2 were analyzed by western blot. $\beta$-actin was used as a loading control. Data are presented as a mean \pm S.E.M. $n=4$. ${ }^{*} p<$ 0.05 versus NT siRNA transfection. All blot and immunofluorescence images shown are representative.

Firmicutes in the gut of obese patients or mice is controversial $^{48-50}$. Our data showed that Firmicutes were hardly changed in HFD-fed mice, which indicates that different results appear to be dependent on age, sex, mouse types, and breeding conditions such as housing density, so it is necessary to conduct further investigations. Therefore, we collected plasma from mice and measured the concentration of $\mathrm{NaB}$ to see if there was actually less $\mathrm{NaB}$ from obese mice. Our results showed lower concentrations of $\mathrm{NaB}$ in obese mice plasma, which may suggest that cross of $\mathrm{NaB}$ into the blood brain barrier can be lower in obese patients. Furthermore, our data revealed that $\mathrm{NaB}$ reduced only BACE1 levels under high cholesterol, which seems to be dependent on cell or treated drug types. We also compared inhibitory effect of SCFAs on amyloidogenesis. Because $\mathrm{NaP}$ is also an HDAC inhibitor, and SCFAs affect cell signaling pathways as GPCR ligands ${ }^{6,51}$, it is necessary to confirm whether $\mathrm{NaB}$ has a stronger inhibitory effect on high cholesterolinduced $A \beta$ secretion. In our results, only $\mathrm{NaB}$ was found to affect a reduction of BACE1 levels. Therefore, we hypothesized that $\mathrm{NaB}$ inhibits BACE1-dependent $\mathrm{A} \beta$ accumulation caused by high cholesterol.

$\mathrm{NaB}$ is absorbed in cells and acts as an HDAC inhibitor and also well known as a GPCR ligand, especially Goi, influencing the cellular signal transduction. Actually, a number of studies reported diverse mechanisms of the antioxidant effect of $\mathrm{NaB}$ in various cell types ${ }^{47,52,53}$. Therefore, we investigated whether $\mathrm{NaB}$ acts as an HDAC inhibitor or a GPCR ligand to inhibit amyloidogenesis caused by high cholesterol. Our results suggest that $\mathrm{NaB}$ acts as an HDAC inhibitor, reducing high-cholesterol- induced amyloidogenesis. Although previous studies suggested that $\mathrm{NaB}$ has the strongest antioxidant effect among SCFAs, comparative experiment was performed by comparing the antioxidant effect of SCFAs [Supplementary Fig. S10]. There is no study on why they have same function but different effects, but this study will help to identify the mechanisms of inhibitory effect of $\mathrm{NaB}$ on $\mathrm{A} \beta$ secretion.

A number of studies described mtROS as major source of excessive ROS, when various cells including neuronal cells are exposed to lethal conditions such as high cholesterol or high glucose ${ }^{54,55}$. Recently, however, there is an increasing number of studies describing the major sources of pathological oxidative stress as NOX family in the CNS, such as microglia and astrocytes as well as neurons ${ }^{20}$. Thus, the major ROS sources appear to be dependent on neuronal cell types or concentrations, times, and types of treatment drug, so further studies are needed. The major ROS source of SK-N-MC cells in hypercholesterolemic environment is not known. Our results indicate that excessive ROS of cells caused by high cholesterol are mainly produced by NOX2. We thereby demonstrated that $\mathrm{NaB}$ prevents excessive ROS through reduction of NOX2 levels. In other words, the effect of $\mathrm{NaB}$ on neurons under high cholesterol is to suppress NOX2 levels, which leads to reduction of ROS formation.

It has been reported that the expression levels of antioxidant enzymes are increased as a protective effect against oxidative stress, whereas oxidative damage such as hyperglycemia inhibits NRF2 pathway, which makes cells vulnerable to oxidative stress ${ }^{56}$. Consistently, our results showed that NRF2 activity was reduced under high 
$\mathbf{a}$

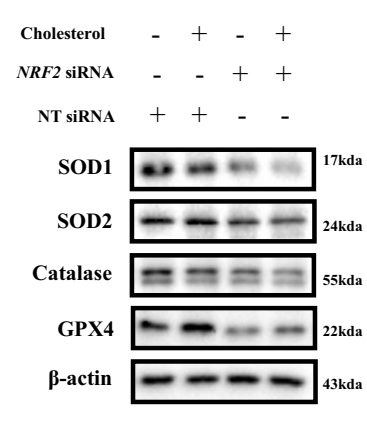

c

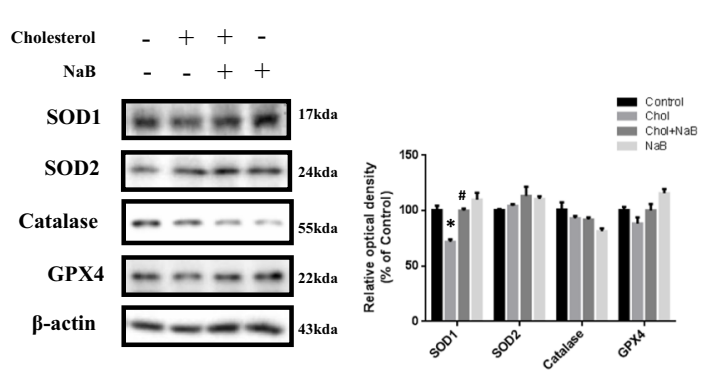

e

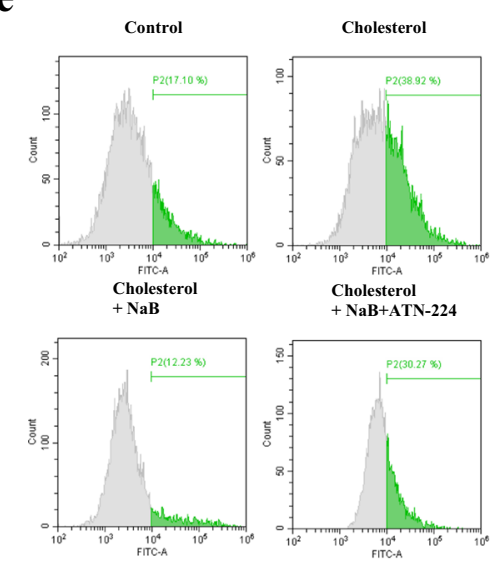

g

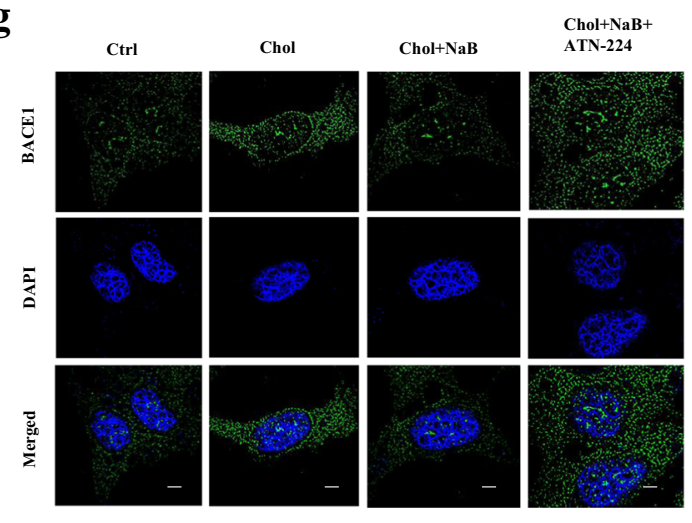

b
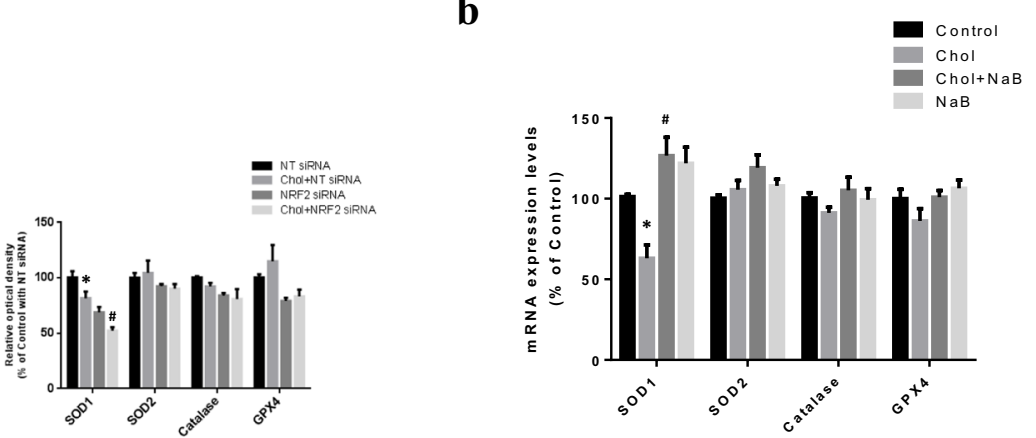

d

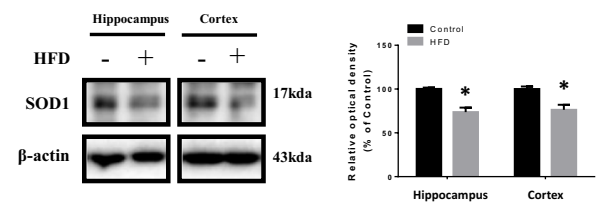

f
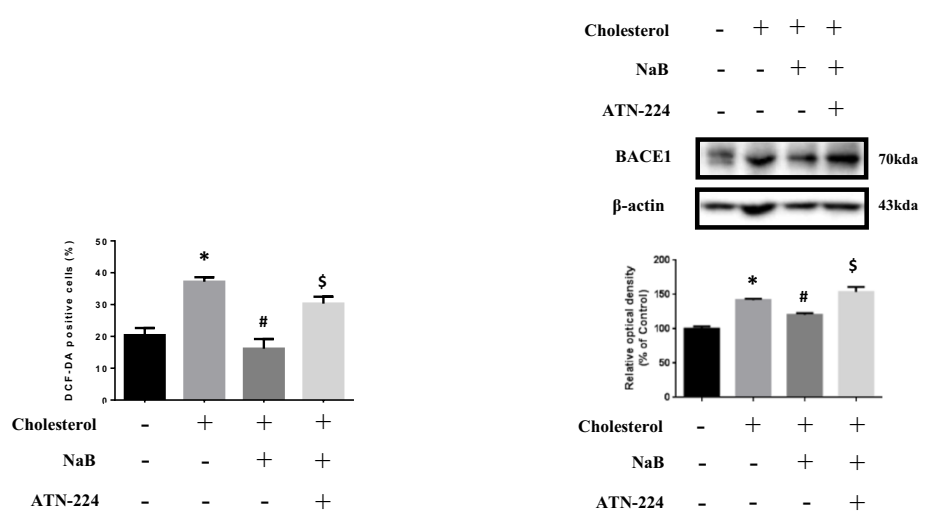

Cholesterol - +++

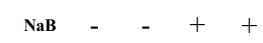

ATN-224 - $-\quad-\quad+$

h
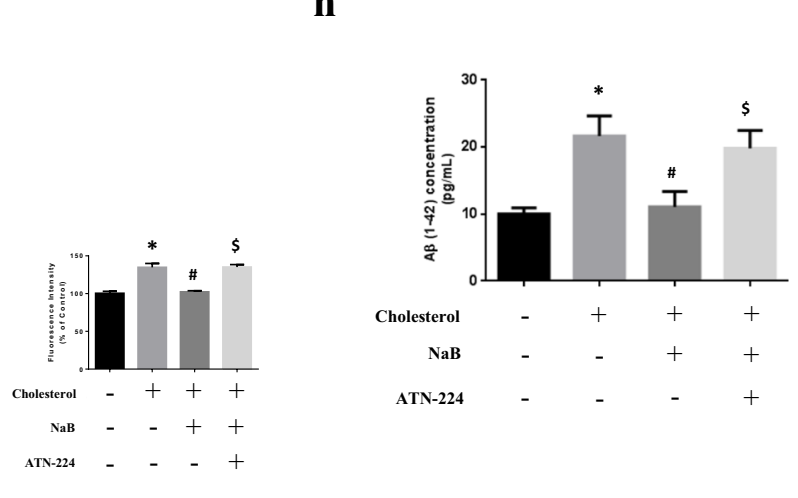

Fig. 6 (See legend on next page.) 
(see figure on previous page)

Fig. 6 Effect of NaB on high-cholesterol-induced ROS generation, BACE1 expression, and A $\beta$ accumulation through SOD1 expression. a SK$\mathrm{N}-\mathrm{MC}$ cells were transfected with NRF2 siRNA or NT siRNA for $24 \mathrm{~h}$, and pretreated with NaB for 30 min prior to treatment of high cholesterol for $24 \mathrm{~h}$. The expression levels of SOD1, SOD2, catalase, and GPX4 were analyzed by western blot. $\beta$-actin was used as a loading control. $n=3 .{ }^{*} p<0.05$ versus control with NT siRNA transfection, ${ }^{*} p<0.05$ versus high cholesterol treatment with NT siRNA transfection. b Cells were pretreated with NaB for $30 \mathrm{~min}$ prior to treatment of high cholesterol for $24 \mathrm{~h}$. mRNA expression levels of SOD1, SOD2, catalase, and GPX4 were analyzed by quantitative realtime PCR. Data were normalized by the ACTB mRNA expression levels. $n=4 .{ }^{*} p<0.05$ versus control, ${ }^{\#} p<0.05$ versus high cholesterol treatment. c Cells were pretreated with $\mathrm{NaB}$ for 30 min prior to treatment of high cholesterol for $24 \mathrm{~h}$. The expression levels of SOD1, SOD2, catalase, and GPX4 were analyzed by western blot. $\beta$-actin was used as a loading control. $n=4 .{ }^{*} p<0.05$ versus control, ${ }^{*} p<0.05$ versus high cholesterol treatment. d Brains of 20 weeks mice fed with HFD for 14 weeks were extracted. The expression levels of SOD1 were analyzed by western blot. $\beta$-actin was used as a loading control. $n=3 .{ }^{*} p<0.05$ versus control. e Cells were pretreated with NaB and ATN2224 $(4 \mu \mathrm{M})$ for 30 min prior to treatment of high cholesterol for $72 \mathrm{~h}$ where ROS with DCF-DA was measured by flowcytometer. Total cell counts $=1.0 \times 10^{4}$ cells. $n=4$. $\mathbf{f}$ Cells were pretreated with $\mathrm{NaB}$ and ATN-224 for $30 \mathrm{~min}$ prior to treatment of high cholesterol for $24 \mathrm{~h}$. The expression levels of BACE1 were analyzed by western blot. $\beta$-actin was used as a loading control. $n=4$. g Cells were pretreated with NaB and ATN-224 for 30 min prior to treatment of high cholesterol for $24 \mathrm{~h}$ and immunostained with BACE1 antibody. Scale bars are $8 \mu \mathrm{m}$ (magnification, $\times 1,000$ ). $n=3$. H Cells were pretreated with NaB and ATN-224 for 30 min prior to treatment of high cholesterol for $72 \mathrm{~h}$. A $\mathrm{B}$ concentration of medium samples was detected by using ELISA kit. Data are presented as a mean \pm S.E.M. $n=4$. ${ }^{*} p<0.05$ versus control, ${ }^{*} p<0.05$ versus high cholesterol treatment, ${ }^{\$} p<0.05$ versus treatment of high cholesterol and $\mathrm{NaB}$. All blot and immunofluorescence images shown are representative.

cholesterol, but $\mathrm{NaB}$ increased the nuclear translocation of NRF2. However, the specific mechanisms of antioxidant effect of $\mathrm{NaB}$ under high cholesterol are not known yet. In our results, p21 levels were decreased by high cholesterol and recovered by $\mathrm{NaB}$, and p21/NRF2 colocalization was also increased, which leads to NRF2 stabilization. However, p21 is a typical cyclindependent kinase inhibitor, which is an apoptosis marker or apoptosis inhibition marker ${ }^{57}$. In addition, there are studies that $\mathrm{NaB}$ contributes to protecting neurons from cell death but adverse studies that $\mathrm{NaB}$ induces apoptosis in Caco- $2^{58,59}$. Therefore, we demonstrated that increased cell death by high cholesterol was reduced by $\mathrm{NaB}$, which means that increased p21 levels by $\mathrm{NaB}$ do not induce apoptosis [Supplementary Fig. S11]. Although previous studies reported that both p53 and Sp1 are critical transcription factors of $\mathrm{p} 21^{43}$, our results showed that increased p21 levels by $\mathrm{NaB}$ are due to $\mathrm{Sp} 1$, not p53 [Supplementary Fig. S12].

NRF2 is a transcription factor that plays an antiinflammatory role and main function of NF- $\mathrm{kB}$ is to regulate the inflammatory response ${ }^{60,61}$. As we referred to studies that NRF2 pathway inhibits NF- $\mathrm{kB}$ activation or an increase in NRF2 activity prevents NF- $\mathrm{BB}$ activation and even activation of NF- $\mathrm{KB}$ affects NRF2/Keap1/ARE signaling pathway ${ }^{45,60,62}$, we need to investigate the crosstalk between two transcription factors, especially under high cholesterol. Our results showed that NRF2 knockdown enhanced NF- $\mathrm{KB}$ activation, followed by increased NOX2 levels. Therefore, considering the references and our results, we suggest that NRF2 stabilization by $\mathrm{NaB}$ suppresses NOX2 levels by weakening NF- $\kappa \mathrm{B}$ activation. However, previous study reported that full transcriptional activity of NF- $\mathrm{kB}$ is regulated by acetylation at lysine $310^{63}$. Although $\mathrm{NaB}$ may directly affects $\mathrm{NF}-\kappa \mathrm{B}$ activation as an HDAC inhibitor, $\mathrm{NaB}$ seems to indirectly influence NF- $\mathrm{B}$ activation via NRF2 stabilization, considering our results showing the correlation between NRF2 and NF- $\mathrm{kB}$. However, the specific mechanism by which $\mathrm{NaB}$ modulates NF- $\mathrm{kB}$ activity needs further study.

In general, previous studies described regulation of transcription factors activation through phosphorylation of transcription factors ${ }^{64,65}$. However, the nuclear translocation of several transcription factors is known to be affected by acetylation, because the acetylation influences stability of transcription factors or protein-protein interaction $^{66}$. However, the activity of transcription factors may be increased or decreased by acetylation, which depends on types of transcription factors or cells ${ }^{44,67,68}$. Although there is no report that regulation of Sp1 acetylation affects $\mathrm{Sp} 1$ activation, our data demonstrated that the acetylation and nuclear translocation of $\mathrm{Sp} 1$ decreased by high cholesterol were recovered by $\mathrm{NaB}$. Thus, these findings suggested that $\mathrm{NaB}$ increases p21 levels by increasing nuclear translocation of Sp1 through regulation of Sp1 acetylation, and eventually induces NRF2 stabilization by contributing to p21/NRF2 colocalization, which is the correlation of NRF2, Sp1, and p21. Subsequently, our data showed that SOD1 of antioxidant enzymes was decreased under high cholesterol and recovered by $\mathrm{NaB}$, but the others were not changed. Alteration of only SOD1 levels seems to be dependent on cell or treated drug types. Therefore, these results suggest that high cholesterol-induced ROS are eliminated by upregulating SOD1 levels via NRF2 stabilization by $\mathrm{NaB}$, which is ultimately to prevent high cholesterol-induced amyloidogenesis.

Taken together, we first revealed the mechanisms of dual effects of $\mathrm{NaB}$ on neuronal cells under high cholesterol. Our study demonstrated that $\mathrm{NaB}$ protects SK-N$\mathrm{MC}$ cells exposed to high cholesterol environment by 


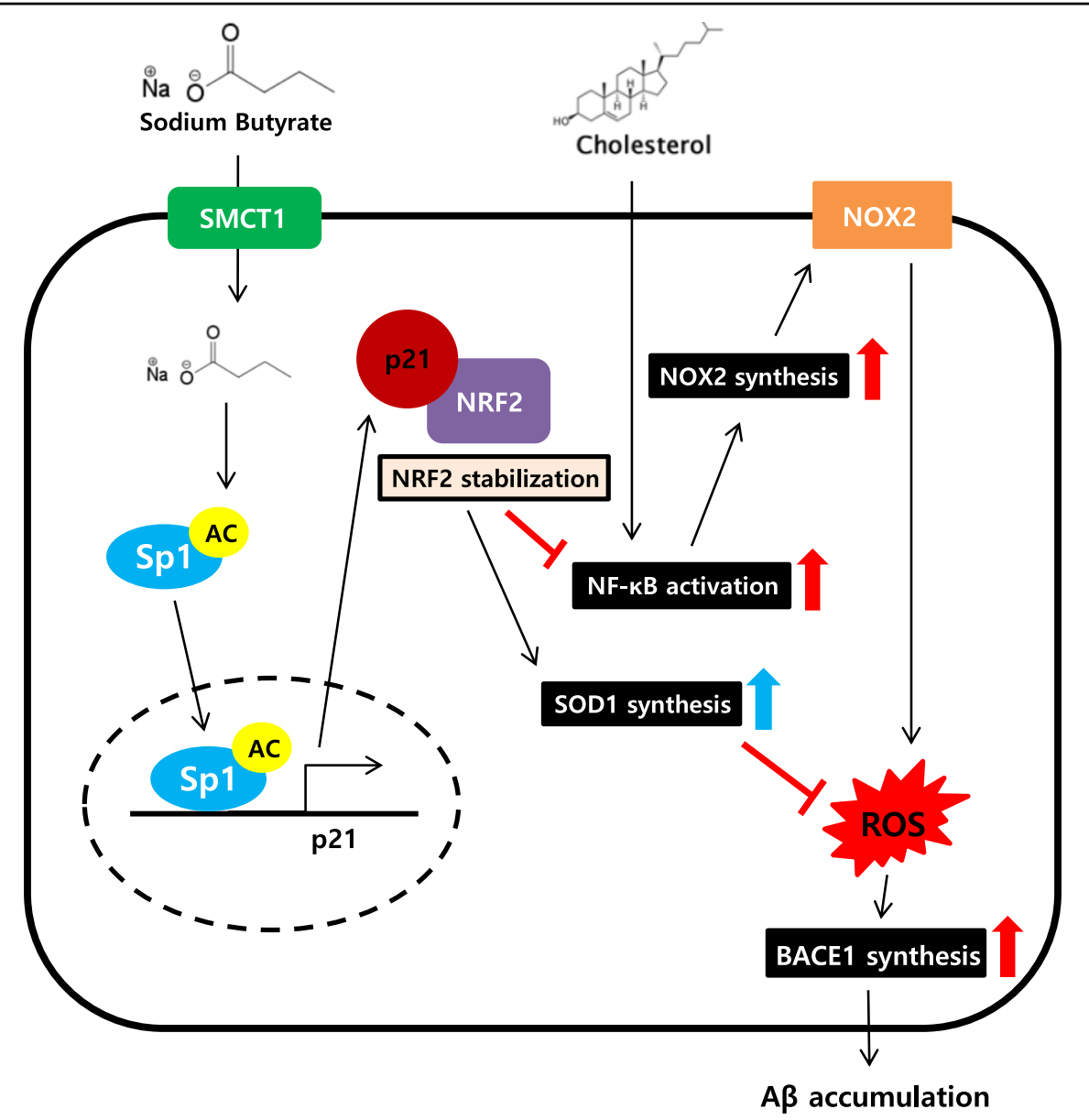

Fig. 7 A hypothetical model for inhibition of high cholesterol-induced neuronal amyloidogenesis by the dual effects of NaB. NaB prevents high cholesterol-induced excessive ROS in SK-N-MC cells through the dual effects of NaB. NaB leads to NRF2 stabilization, followed by suppression of NOX2 levels via reducing the nuclear translocation of NF-KB and upregulation of SOD1 levels. NaB ultimately inhibits BACE1-dependent high cholesterol-induced neuronal amyloidogenesis by modulating NRF2 stabilization-mediated ROS levels.

modulating NRF2 stabilization-mediated ROS levels. In conclusion, we showed that $\mathrm{NaB}$ inhibits BACE1dependent high cholesterol-induced neuronal amyloidogenesis by suppressing NOX2 levels and upregulating SOD1 levels via p21/NRF2 pathway (Fig. 7). Furthermore, it suggests that $\mathrm{NaB}$ can be a therapeutic strategic candidate to $\mathrm{AD}$ patients caused by obesity, because obese patients who are most likely to get $\mathrm{AD}$ showed reduced butyrate-producing bacteria in the microbiota composition.

\section{Materials and methods Materials}

The human neuroblastoma cell line SK-N-MC was acquired from Korean Cell Line Bank (Seoul, Korea, RRID: CVCL_1398). Fetal bovine serum (FBS) and Dulbecco's modified eagle medium (DMEM) were purchased from Hyclone (Logan, UT, USA). Antibiotics and serum replacement (SR) were acquired from Gibco (Grand
Island, NY, USA). The antibodies of $\beta$-actin (sc-47778), Lamin A/C (sc-376248), GPX4 (sc-166570), catalase (sc271803), NF-kB (sc-8008), BACE1 (sc-33711), and PSEN1 (sc-365450) were purchased from Santa Cruz Biotechnology (Santa Cruz, CA, USA). The antibodies of APP (ab32136), BACE1 (ab2077), Sp1 (ab227383), NRF2 (ab89443), and A $\beta$ (ab2530) were purchased from Abcam (Cambridge, England). The antibody of NOX2 (611414) was purchased from BD bioscience. The antibody of SOD1 (CSB-PA02864A0Rb) was purchased from CusaBio (Houston, TX, USA) and SOD2 (06-984) was purchased from EMD Millipore (Burlington, MA, USA). The antibody of Sp1 (H00006667-MO3) was purchased from Novus (Littleton, CO, USA) and Acetylated-Lysine (9441 S) was purchased from Cell Signaling Technology (Danvers, MA, USA). The antibodies of p53 (PA5-27822) and p21 (MA5-14949) were purchased from Thermo Fisher and TurboFect, human or mouse $A \beta(1-42)$ ELISA kit, CM-H2DCFDA, MitoSOX ${ }^{\mathrm{TM}}$ Red, and RNAlater ${ }^{\mathrm{TM}}$ 
solution were also obtained from Thermo Fisher (Waltham, MA, USA). ATN-224 was purchased from CSNpharm and Bay11-7082 was purchased from Calbiochem. Paraformaldehyde (PFA) was purchased from Lugen (Bucheon, Korea). EzSubcell ${ }^{\mathrm{TM}}$ subcellular fractionation kit was acquired from Atto (Tokyo, Japan). Sodium acetate was purchased from FUJIFILM Wako Pure Chemical Corporation. NAC, Vas2870, mitotempo, sodium butyrate, sodium propionate, butyric acid, acrylic acid, mphosphoric acid, propyl formate, cholesterol-water soluble, DAPI, Triton X-100, ibuprofen, and PTX were purchased from Sigma Chemical Company (St. Louis, MO, USA). mRNA primers for APP, BACE1, PSEN1, NOX1, NOX2, NOX3, NOX4, SOD1, SOD2, catalase, GPX4, and $A C T B$ were purchased from Cosmo Genetech (Seoul, Korea). SMCT1, NRF2, BACE1, NOX2, SOD1, p65, Sp1, $p 21$ siRNA were acquired from Bioneer (Daejeon, Korea). NT siRNA was purchased from Dharmacon (Lafayette, CO, USA).

\section{Culture of SK-N-MC cells}

SK-N-MC cells were cultured without a feeder layer in high-glucose Dulbecco's Modified Eagle Medium (DMEM), supplemented with $10 \%$ FBS and $1 \%$ antibiotic-antimycotic solution at $37^{\circ} \mathrm{C}$ with $5 \% \mathrm{CO}_{2}$. Cells were grown in $60,100 \mathrm{~mm}$ diameter culture dishes, or 12-, 96-well plates in an incubator maintained at $37^{\circ} \mathrm{C}$ with $5 \% \mathrm{CO}_{2}$. When the cells reach approximately $60 \%$ confluency, the medium was replaced with DMEM supplemented with $1 \%$ antibiotic-antimycotic solution and $2 \%$ of SR $24 \mathrm{~h}$ before the experiments. Cells were maintained in 2\% SR in DMEM containing $1 \%$ antibiotics and agents such as cholesterol, $\mathrm{NaB}$, and ibuprofen.

\section{Preparation of brain tissue of the HFD-induced obese mouse model}

To induce obesity through a diet, 6-week-old male ICR mice were fed either a regular chow diet or a HFD (Rodent Diet with $60 \mathrm{kcal} \%$ Fat and $90 \mathrm{~g}$ Added $\mathrm{NaCl}$ /4057 kcal, Research Diets Inc., New Brunswick, NJ, USA) for 14 weeks. Mice were stabilized in the new environment for 3 days before experimentation. Mice were granted ad libitum access to food and water. The room was maintained under standard environmental conditions $\left(24 \pm 2{ }^{\circ} \mathrm{C}, 12 / 12 \mathrm{~h}\right.$ light/dark cycle with lights on at 07:00 a.m.) Mice were randomly assigned. The experiments were performed according to the "Guide for Animal Experiments" (Edited by the Korean Academy of Medical Sciences) and approved by the Institutional Animal Care and Use Committee (IACUC) of Seoul National University (SNU-180802-2). The brains were removed and post-fixed by $4 \%$ paraformaldehyde in $0.1 \mathrm{M}$ PBS for $12 \mathrm{~h}$. The brain tissues were cryo-protected by overnight infusion with $90 \%$ sucrose, and $30 \mu \mathrm{m}$-thick coronal sections were serially cut using a cryostat (Leica, Deerfield, Germany). The sections were transferred to 6-well plates containing PBS for further processing.

\section{Metagenomic $16 \mathrm{~S}$ rRNA sequencing and data analysis}

Mouse fecal samples were stored in RNAlater ${ }^{\mathrm{TM}}$ solution at $-80^{\circ} \mathrm{C}$ until processing. DNA extractions for $16 \mathrm{~S}$ rRNA analysis were performed by using FastDNA ${ }^{\circledR}$ SPIN Kit for Soil (MP Biomedicals, Solon, CA, USA) based on manufacturer's recommendations. The bacterial $16 \mathrm{~S}$ rRNA V3-V4 region was amplified in accordance with the Illumina $16 \mathrm{~S}$ Metagenomic Sequencing Library Preparation guide (Illumina, CA, USA), using the primers with added adapter overhang sequences: ${ }^{69}$ forward primer: 5'-TCGTCGGCAGCGTCAGATGTGTATAAGAGACA GCCTACGGGNGGCWGCAG -3', reverse primer: 5'- G TCTCGTGGGCTCGGAGATGTGTATAAGAGACAG GACTACHVGGGTATCTAATCC -3'. Also, the MiSeq platform (Illumina, CA, USA) with the $2 \times 300$ bp pairedend sequencing was used for amplicon sequencing through standard Illumina sequencing protocols. A quality control for the raw sequences of each sample was examined using FastQC ${ }^{70}$. Then, QIIME2 (v.2019.4) ${ }^{71}$, a standardized pipeline for $16 \mathrm{~S}$ rRNA sequencing data was processed. The q2-cutadapt plugin $^{72}$ was used for removing the primer sequences and The DADA2 software package $^{73}$ wrapped in QIIME2 was used to quality control including quality filtering, denoising, and used to feature table construction containing amplicon sequence variants (ASVs). After that, the q2-phylogeny plugin was used for generating the phylogenetic tree. Furthermore, alpha diversity ( $\alpha$-diversity) and beta diversity ( $\beta$-diversity) were performed as the core-metrics-phylogenetic in the QIIME2 diversity plugin provides these two types of diversity analysis. For taxonomic annotation, the QIIME2 feature-classifier plugin (classify-sklearn) with a pretrained Naïve Bayes classifier on the GreenGene database (v.13_8 with 99\% similarity operational taxonomic units $(\mathrm{OTUs}))^{74}$ was performed. Finally, the Statistical Analysis of Metagenomic Profiles (STAMP) software ${ }^{75}$ was used for comparing the taxonomic profiling analysis between groups.

\section{Preparation of plasma samples}

All procedures for preparation of samples were performed following the previous report ${ }^{76}$. Hundred microliters of internal standard solution $(150 \mu \mathrm{M}$ acrylic acid, $1500 \mu \mathrm{M}$ m-phosphoric acid) was added to $200 \mu \mathrm{l}$ plasma. Samples were vortexed for $5 \mathrm{~min}$ followed by centrifugation (30 min, 14,916 rpm) and left to solidify the precipitate for $30 \mathrm{~min}$ at $4{ }^{\circ} \mathrm{C}$. Hundred microliters of the clear supernatant was transferred into a new tube and $100 \mu \mathrm{l}$ washed propyl formate was added. Samples were vortexed for $5 \mathrm{~min}$ followed by centrifugation $(10 \mathrm{~min}$, 
$14,916 \mathrm{rpm}$ ) before transferring $50 \mu \mathrm{l}$ of the organic layer into GC vials (Waters, Milford, Massachusetts, United States) for analysis.

\section{GC-MS analysis}

One microliter sample was injected into a straight glass liner, held at $200^{\circ} \mathrm{C}$. Helium $(1 \mathrm{ml} / \mathrm{min})$ was used as carrier gas in TR-FAME $(25 \mathrm{~m} \times 0.32 \mathrm{~mm} \times 0.25 \mu \mathrm{m})$. The initial oven temperature of $60^{\circ} \mathrm{C}$ was maintained for 4 min, then ramped to $130^{\circ} \mathrm{C}$ at $50^{\circ} \mathrm{C} / \mathrm{min}$ and held for $3.7 \mathrm{~min}$ and finally raised to $240^{\circ} \mathrm{C}$ at $30^{\circ} \mathrm{C} / \mathrm{min}$ and held for $10 \mathrm{~min}$. The transfer line and ion source temperature is $250^{\circ} \mathrm{C}$. All samples, standards, and blanks were analyzed randomly at the GC-MS system. Trace 1300 Series gas chromatograph with TSQ Series mass spectrometer (Thermo Fisher, MA, USA) was used. Integrations were performed automatically with Triplus RSH autosampler (Thermo Fisher, MA, USA).

\section{Western blot analysis}

Cells were detached from the 60 or $100 \mathrm{~mm}$ diameter culture dishes with a scraper and gathered by centrifugation $\left(13,200 \mathrm{rpm}, 4^{\circ} \mathrm{C}, 5 \mathrm{~min}\right)$. Harvested cells and brain tissues were lysed by RIPA lysis buffer (ATTO Corporation, Tokyo, Japan) and incubated for $30 \mathrm{~min}$ on ice. The lysates were then cleared by centrifugation $\left(13,200 \mathrm{rpm}, 4{ }^{\circ} \mathrm{C}, 30 \mathrm{~min}\right)$. The protein concentration was measured by the bicinchoninic acid (BCA) assay kit (BioRad, Hercules, CA, USA). Samples containing $10 \mu \mathrm{g}$ of protein were prepared for $8-15 \%$ sodium dodecyl sulfatepolyacrylamide gel electrophoresis (SDS-PAGE) and then transferred to a polyvinylidene fluoride (PVDF) membrane and blocked with 5\% skim milk (Gibco) dissolved in TBST for $40 \mathrm{~min}$. The blocked membranes were washed with TBST and incubated with primary antibody overnight at $4{ }^{\circ} \mathrm{C}$. The membranes were subsequently washed and incubated with HRP-conjugated secondary antibody at room temperature for $2 \mathrm{~h}$. The western blotting bands were visualized by means of chemiluminescence (Bio-Rad, Hercules, CA, USA). Specific bands were detected using a ChemiDoc $^{\mathrm{TM}}$ XRS+System (Bio-Rad, Hercules, CA, USA) and analyzed by using ImageJ software.

\section{Reverse transcription-polymerase chain reaction (RT-PCR) and real-time PCR}

RNA was extracted using MiniBEST Universal RNA Extraction Kit (TaKaRa, Otsu, Shinga, Japan). Reverse transcription was performed using $1 \mu \mathrm{g}$ of RNA with a Maxime RT-PCR PreMix Kit (Intron Biotechnology, Seongnam, Korea) to obtain cDNA. Two microliters of cDNA were then amplified using Quanti NOVA SYBR Green PCR Kits (Qiagen, Hilden, Germany). Real-time quantification of RNA targets was performed in a RotorGene 6000 real-time thermal cycling system (Corbett
Research, NSW, Australia). The reaction mixture $(20 \mu \mathrm{l})$ contained $200 \mathrm{ng}$ of total RNA, $0.5 \mathrm{mM}$ of each primer for the gene of interest, and appropriate amounts of enzymes and fluorescent dyes as recommended by the manufacturer. The real-time PCR was performed as follows: $15 \mathrm{~min}$ at $95^{\circ} \mathrm{C}$ for DNA polymerase activation; $15 \mathrm{~s}$ at $95^{\circ} \mathrm{C}$ for denaturing; and 50 cycles of $15 \mathrm{~s}$ at $94{ }^{\circ} \mathrm{C}, 30 \mathrm{~s}$ at $54{ }^{\circ} \mathrm{C}$, and $30 \mathrm{~s}$ at $72^{\circ} \mathrm{C}$. Data were collected during the extension step, and analysis was performed using the software provided; melting curve analysis was performed to verify the specificity and identity of the PCR products. Normalization of gene expression levels was performed by using the $A C T B$ gene as an endogenous control. Sequences of the primers used are described in Supplementary Table S2.

\section{Detection of intracellular ROS and mitochondrial ROS}

The CM-H2DCFDA and MitoSOX ${ }^{\mathrm{TM}}$ Red were used to measure the intracellular ROS and mitochondrial ROS respectively. Cells were seeded in a 96-well plate at same density $\left(2 \times 10^{3}\right.$ cells per well). Cells were treated with $5 \mu \mathrm{M}$ DCF-DA or $2 \mu \mathrm{M}$ MitoSOX ${ }^{\mathrm{TM}}$ Red and then incubated in an incubator at $37^{\circ} \mathrm{C}$ with $5 \% \mathrm{CO}_{2}$ for $30 \mathrm{~min}$ or $15 \mathrm{~min}$, respectively. Cells were washed twice with PBS and then measured with a luminometer (Victor3; PerkinElmer Inc., Waltham, MA, USA). The fluorescence intensity of CM-H2DCFDA was measured with a luminometer at an excitation and emission wavelength of 485 and $535 \mathrm{~nm}$ and MitoSOX ${ }^{\mathrm{TM}}$ Red was measured at an excitation and emission wavelength of 530 and $580 \mathrm{~nm}$.

\section{Flow cytometry}

Cells were seeded in 12-well culture dishes. When cells were at $60 \%$ confluence, medium was replaced with $2 \%$ SR in DMEM containing $1 \%$ antibiotics for $24 \mathrm{~h}$. Cells were stained with $5 \mu \mathrm{M}$ DCF-DA for $30 \mathrm{~min}$ or $2 \mu \mathrm{M}$ MitoSox ${ }^{\mathrm{TM}}$ Red for $15 \mathrm{~min}$. Cells were washed with PBS two times and treated with a $0.05 \%$ trypsin and $0.5 \mathrm{mM}$ EDTA solution to detach cells from the dish for $3 \mathrm{~min}$. Cells were centrifuged at $3000 \mathrm{rpm}$ for $5 \mathrm{~min}$. DCF-DA or MitoSOX $^{\mathrm{TM}}$ Red staining were determined via flow cytometry (CytoFlex; Beckman Coulter, Fullerton, CA, USA).

\section{Nuclear fractionation}

To prepare the cytosolic and nuclear-fractionized samples, the EzSubcell ${ }^{\mathrm{TM}}$ subcellular fractionation/extraction kit was used. All procedures of the subcellular fractionation assay followed manufacturer's protocol. Nuclear NF- $\mathrm{kB}, \mathrm{NRF} 2$, p53, and Sp1 expression levels in nuclearfractionized samples were normalized by Lamin A/C expression levels.

\section{siRNA transfection}

When cells were grown until $60 \%$ confluence, cells were transfected with SMCT1, NRF2, BACE1, NOX2, SOD1, 
$p 65, S p 1, p 21$ siRNA, and nontargeting (NT) siRNA for $12 \mathrm{~h}$ using TurboFectTM transfection reagent in $2 \%$ SR in DMEM containing $1 \%$ antibiotics. After $12 \mathrm{~h}$ of incubation, the culture media were replaced with $2 \%$ SR in DMEM containing $1 \%$ antibiotics and the cells were maintained for $24 \mathrm{~h}$. The concentration of each transfected siRNA was $25 \mathrm{nM}$. The sequences of siRNAs used are described in Supplementary Table S3.

\section{Immunocytochemistry}

Cells were washed with PBS on a confocal dish. Cells were fixed with $4 \%$ paraformaldehyde (PFA) for $10 \mathrm{~min}$ and then, incubated in $0.1 \%$ Triton X-100 for membrane permeabilization for $10 \mathrm{~min}$. Cells were incubated with $5 \%$ normal goat serum in PBS for $30 \mathrm{~min}$ and with primary antibodies overnight at $4{ }^{\circ} \mathrm{C}$. Next, Cells were washed with PBS three times and incubated with Alexa Fluor ${ }^{\mathrm{TM}} 488$ or 555-conjugated secondary antibodies for $2 \mathrm{~h}$ at room temperature. Immunofluorescence images were obtained by a super-resolution radial fluctuations (SRRF) imaging system (Andor Technology, Belfast, UK) ${ }^{77}$.

\section{Immunohistochemistry}

Tissues were deparaffinized with xylene and 100, 95, 70, and $50 \%$ ethanol. For peroxide inactivation, the tissues were then incubated on $3 \% \mathrm{H}_{2} \mathrm{O}_{2}$ in methanol and washed with PBS. For permeabilization, the tissues were incubated in PBS containing $0.5 \%$ Triton $\mathrm{x} 100$ for $15 \mathrm{~min}$, washed with PBS, and incubated with 5\% NGS in PBS for $30 \mathrm{~min}$. The tissues were labeled with 5\% NGS in PBS containing APP, BACE1, and $\mathrm{A} \beta$ antibodies at a ratio of 1:100 for $2 \mathrm{~h}$, followed by secondary antibody and PI in 1:100 ratio for $1 \mathrm{~h}$ at room temperature. After washing with PBS for $15 \mathrm{~min}$, images were obtained by using FluoView $^{\mathrm{TM}} 300$ confocal microscope (Olympus).

\section{A $\beta$ ELISA}

The A $\beta$ (1-42) concentration level in medium sample was measured by commercial enzyme-linked immunosorbent assay (ELISA) kits. Medium samples were collected and centrifugated at 13,200 rpm for $5 \mathrm{~min}$ and the supernatant samples were collected as ELISA samples. All procedures of the A $\beta$ ELISA assay were performed according to the manufacturer's protocol.

\section{Water soluble tetrazolium salt (WST-1) assay}

WST-1 assay was used for determining cell proliferation and cell viability in vitro model. All procedures of the assay were conducted according to the manufacturer's protocol. Cells were seeded in 96 -well plate $\left(2 \times 10^{3}\right.$ cells per well). EZ-Cytox ${ }^{\mathrm{TM}}$ reagent was added to each well and the cells were incubated for $1 \mathrm{~h}$ in $5 \% \mathrm{CO}_{2}$ incubator at $37^{\circ} \mathrm{C}$. The absorbance of each sample was measured at a wavelength of $450 \mathrm{~nm}$ by using a microplate reader.

\section{Trypan-blue cell viability assay}

The SK-N-MC cells were washed with PBS, and then incubate with a $0.05 \%$ trypsin and $0.5 \mathrm{mM}$ EDTA solution to detach the cells. Trypsin inhibitor (PBS with 10\% FBS) was added to quench trypsin. The cell suspension solution was centrifugated 3,000 rpm for $5 \mathrm{~min}$. The cell pellet was suspended with $0.4 \%$ trypan blue in PBS to stain the dead cells. Ten microliters was dispensed on the cell counting chamber slides. Trypan-blue stained or unstained cells were counted by using a Countess II FL (Thermo Fisher, MA, USA).

\section{Statistical analysis}

For the reliability of the experiment, we randomized through each experiment and performed the experiments. All samples were randomly assigned following simple randomization procedures (computerized random numbers) to one of two treatment groups. All experiments in vitro and animal tests were performed and assessed in a blinded-fashion. The number of replicates was 3-4 throughout all experiments. We used SigmaPlot (version 12.0, Systat Software Inc.) to choose the appropriate sample size: The sample size is calculated considering the power (1-beta) for statistical validity and Difference in Means, Standard Deviation of the expected result. We established the inclusion/exclusion criteria to derive more accurate and reliable data. Because some outliers may have significant something, we valued to meet the pretest data. Therefore, we excluded the specific confounding variables compared with pretest data. Quantitative data are presented as the mean \pm standard error of the mean (S.E.M). Statistical analysis was conducted by using GraphPad Prism Version 6.0 (GraphPad Inc., San Diego, CA, USA). All data achieved normality and were analyzed by parametric statistics through the GraphPad Prism Version 6.0. The variation estimate of the data acquired through the pretest was obtained, and then it was confirmed that the normal distribution was achieved. Based on this, the experiments were performed, and the data actually satisfied the normality. Statistical differences between more than two groups and within groups variance were assessed by using analysis of variance (ANOVA). One-way ANOVA (with Dunnett's multiple comparison test) or Two-way ANOVA (with Tukey's multiple comparison test) were used for analyzing the differences among groups. The student's $t$-test was conducted for comparing the means of treatment groups with that of the control group. $p<0.05$ was considered statistically significant.

\section{Acknowledgements}

This research was supported by National R\&D Program through the National Research Foundation of Korea (NRF) funded by the Ministry of Science and ICT (NRF-2020R1 A2B5B02002442, NRF-2017M3A9F3046543) and BK21 PLUS

Program for Creative Veterinary Science Research. 


\section{Author details}

'Department of Veterinary Physiology, College of Veterinary Medicine, Research Institute for Veterinary Science, and BK21 PLUS Program for Creative Veterinary Science Research, Seoul National University, Seoul 08826, Republic of Korea. '2Laboratory of Veterinary Physiology, College of Veterinary Medicine, Chungbuk National University, Cheongju, Chungbuk 28644, South Korea. ${ }^{3}$ Institute for Stem Cell \& Regenerative Medicine (ISCRM), Chungbuk National University, Cheongju 28644 Chungbuk, Korea. ${ }^{4}$ Department of Biomedical Science and Engineering, Gwangju Institute of Science and Technology (GIST), Gwangju, Korea. ${ }^{5}$ Genome and Company, Pangyo-ro 253, Bundang-gu. Seoungnam-si, Gyeonggi-do 13486, Korea

\section{Conflict of interest}

The authors declare that they have no conflict of interest.

\section{Publisher's note}

Springer Nature remains neutral with regard to jurisdictional claims in published maps and institutional affiliations.

Supplementary Information accompanies this paper at (https://doi.org/ 10.1038/s41419-020-2663-1).

Received: 7 November 2019 Revised: 14 May 2020 Accepted: 3 June 2020 Published online: 18 June 2020

\section{References}

1. Westfall, S. et al. Microbiome, probiotics and neurodegenerative diseases: deciphering the gut brain axis. Cell Mol. Life Sci. 74, 3769-3787 (2017).

2. Li, Z., Zhu, H., Zhang, L. \& Qin, C. The intestinal microbiome and AD: a review. Anim. Model Exp. Med. 1, 180-188 (2018).

3. Tremlett, H., Bauer, K. C., Appel-Cresswell, S., Finlay, B. B. \& Waubant, E. The gut microbiome in human neurological disease: a review. Ann. Neurol. 81, 369-382 (2017).

4. Zhao, Y., Cong, L., Jaber, V. \& Lukiw, W. J. Microbiome-derived lipopolysaccharide enriched in the perinuclear region of AD brain. Front. Immunol. 8 1064 (2017).

5. Jakobsdottir, G., Xu, J., Molin, G., Ahrne, S. \& Nyman, M. High-fat diet reduces the formation of butyrate, but increases succinate, inflammation, liver fat and cholesterol in rats, while dietary fibre counteracts these effects. PLOS ONE 8, e80476 (2013).

6. Lu, Y. et al. SCFAs prevent HFD-induced obesity in mice by regulating GPCR and gut microbiota. Sci. Rep. 6, 37589 (2016).

7. Govindarajan, N., Agis-Balboa, R. C., Walter, J., Sananbenesi, F. \& Fischer, A Sodium butyrate improves memory function in an AD mouse model when administered at an advanced stage of disease progression. J. Alzheimers Dis. 26, 187-197 (2011).

8. Canani, R. B. et al. Potential beneficial effects of butyrate in intestinal and extraintestinal diseases. World J. Gastroenterol. 17, 1519-1528 (2011).

9. McNabney, S. M. \& Henagan, T. M. SCFAs in the colon and peripheral tissues: a focus on butyrate, colon cancer, obesity and insulin resistance. Nutrients $\mathbf{9}$, https://doi.org/10.3390/nu9121348 (2017).

10. Hong, J. et al. Butyrate alleviates HFD-induced obesity through activation of adiponectin-mediated pathway and stimulation of mitochondrial function in the skeletal muscle of mice. Oncotarget 7, 56071-56082 (2016).

11. Davie, J. R. Inhibition of histone deacetylase activity by butyrate. J. Nutr. 133 2485s-2493s (2003)

12. Alford, S., Patel, D., Perakakis, N. \& Mantzoros, C. S. Obesity as a risk factor for AD: weighing the evidence. Obes. Rev. 19, 269-280 (2018).

13. Pugazhenthi, S., Qin, L. \& Reddy, P. H. Common neurodegenerative pathways in obesity, diabetes, and AD. Biochim. Biophys. Acta Mol. Basis Dis. 1863 1037-1045 (2017)

14. Profenno, L. A., Porsteinsson, A. P. \& Faraone, S. V. Meta-analysis of AD risk with obesity, diabetes, and related disorders. Biol. Psychiatry 67, 505-512 (2010).

15. Klop, B., Elte, J. W. \& Cabezas, M. C. Dyslipidemia in obesity: mechanisms and potential targets. Nutrients 5, 1218-1240 (2013).

16. Kivipelto, M. \& Solomon, A. Cholesterol as a risk factor for AD-epidemiological evidence. Acta Neurol. Scand. Suppl. 185, 50-57 (2006).
17. Wang, C. et al. Cholesterol enhances colorectal cancer progression via ROS elevation and MAPK signaling pathway activation. Cell Physiol. Biochem. 42 729-742 (2017).

18. Sun, J. H., Yu, J. T. \& Tan, L. The role of cholesterol metabolism in AD. Mol Neurobiol. 51, 947-965 (2015).

19. Bae, Y. S., Oh, H., Rhee, S. G. \& Yoo, Y. D. Regulation of ROS generation in cell signaling. Mol. Cells 32, 491-509 (2011).

20. Nayernia, Z., Jaquet, V. \& Krause, K. H. New insights on NOX enzymes in the central nervous system. Antioxid. Redox Signal 20, 2815-2837 (2014).

21. David, J. A., Rifkin, W. J., Rabbani, P. S. \& Ceradini, D. J. The NRF2/Keap1/ARE Pathway and oxidative stress as a therapeutic target in type II diabetes mellitus. J. Diabetes Res. 2017, 4826724 (2017).

22. Hayes, J. D. \& Dinkova-Kostova, A. T. The NRF2 regulatory network provides an interface between redox and intermediary metabolism. Trends Biochem. Sci. 39. 199-218 (2014)

23. Matough, F. A., Budin, S. B., Hamid, Z. A., Alwahaibi, N. \& Mohamed, J. The role of oxidative stress and antioxidants in diabetic complications. Sultan Qaboos Univ. Med. J. 12, 5-18 (2012).

24. Dong, W. et al. Sodium butyrate activates NRF2 to ameliorate diabetic nephropathy possibly via inhibition of HDAC. J. Endocrinol. 232, 71-83 (2017).

25. Chen, W. et al. Direct interaction between Nrf2 and p21(Cip1MAF1) upregulates the Nrf2-mediated antioxidant response. Mol. Cell 34, 663-673 (2009).

26. Crim, K. C. et al. Upregulation of p21Waf1/Cip1 expression in vivo by butyrate administration can be chemoprotective or chemopromotive depending on the lipid component of the diet. Carcinogenesis 29, 1415-1420 (2008).

27. Lee, S. et al. Assessment of cognitive impairment in a mouse model of HFDinduced metabolic stress with touchscreen-based automated battery system. Exp. Neurobiol. 27, 277-286 (2018).

28. Duffy, C. M., Hofmeister, J. J., Nixon, J. P. \& Butterick, T. A. HFD increases cognitive decline and neuroinflammation in a model of orexin loss. Neurobiol. Learn. Mem. 157, 41-47 (2019).

29. McLean, F. H. et al. Rapid and reversible impairment of episodic memory by a HFD in mice. Sci. Rep. 8, 11976 (2018).

30. Waheed Roomi, M. Kalinovsky, T, Roomi, N. W Niedzwiecki, A \& Rath, M Inhibition of the SK-N-MC human neuroblastoma cell line in vivo and in vitro by a novel nutrient mixture. Oncol. Rep. 29, 1714-1720 (2013).

31. Kim, J. Y. et al. Palmitic acid-BSA enhances A $\beta$ production through GPR40mediated dual pathways in neuronal cells: Involvement of the Akt/mTOR/HIF1a and Akt/NF-KB pathways. Sci. Rep. 7, 4335 (2017)

32. Choi, G. E. et al. Membrane-associated effects of glucocorticoid on BACE1 upregulation and $A \beta$ generation: Involvement of lipid raft-mediated CREB activation. J. Neurosci. 37, 8459-8476 (2017)

33. Petrov, V. A. et al. Analysis of gut microbiota in patients with Parkinson's disease. Bull. Exp. Biol. Med. 162, 734-737 (2017)

34. Goodrich, J. K. et al. Conducting a microbiome study. Cell 158, 250-262 (2014).

35. Baxter, N. T. et al. Dynamics of human gut microbiota and SCFAs in response to dietary interventions with three fermentable fibers. mBio 10, e02566-02518 (2019).

36. Zoubair, B. et al. Evaluation of ibuprofen acid effect on oxidative stressed mice J. Chem. Pharm. Res. 8, 382-388 (2016).

37. Martin, P. M. et al. Identity of SMCT1 (SLC5A8) as a neuron-specific $\mathrm{Na}^{+}$-coupled transporter for active uptake of L-lactate and ketone bodies in the brain. J. Neurochem. 98, 279-288 (2006).

38. Manea, S. A., Constantin, A., Manda, G., Sasson, S. \& Manea, A. Regulation of NOX enzymes expression in vascular pathophysiology: focusing on transcription factors and epigenetic mechanisms. Redox Biol. 5, 358-366 (2015).

39. Cooney, S. J., Bermudez-Sabogal, S. L. \& Byrnes, K. R. Cellular and temporal expression of NOX isotypes after brain injury. J. Neuroinflammation 10,155 (2013).

40. Hernandes, M. S. \& Britto, L. R. NOX and neurodegeneration. Curr. Neuropharmacol. 10, 321-327 (2012)

41. de la Monte, S. M. \& Wands, J. R. Molecular indices of oxidative stress and mitochondrial dysfunction occur early and often progress with severity of AD. J. Alzheimers Dis. 9, 167-181 (2006).

42. Auer, S. et al. The human NOX, NOX4, regulates cytoskeletal organization in two cancer cell lines, HepG2 and SH-SY5Y. Front. Oncol. 7, 111 (2017).

43. Koutsodontis, G., Tentes, I., Papakosta, P., Moustakas, A. \& Kardassis, D. Sp1 plays a critical role in the transcriptional activation of the human cyclin-dependent kinase inhibitor p21(WAF1/Cip1) gene by the p53 tumor suppressor protein. J. Biol. Chem. 276, 29116-29125 (2001). 
44. Kundumani-Sridharan, V. et al. Novel interactions between NFATc1 (Nuclear Factor of Activated T cells C1) and STAT-3 (Signal Transducer and Activator of Transcription-3) mediate GPCR agonist, thrombin-induced biphasic expression of cyclin D1, with first phase influencing cell migration and second phase directing cell proliferation. J. Biol. Chem. 287, 22463-22482 (2012).

45. Ganesh Yerra, V., Negi, G., Sharma, S. S. \& Kumar, A. Potential therapeutic effects of the simultaneous targeting of the NRF2 and NF-KB pathways in diabetic neuropathy. Redox Biol. 1, 394-397 (2013).

46. Li, W. et al. Activation of NRF2-antioxidant signaling attenuates NF-KB -inflammatory response and elicits apoptosis. Biochem. Pharm. 76, 1485-1489 (2008).

47. Jahns, F. et al. Butyrate modulates antioxidant enzyme expression in malignant and non-malignant human colon tissues. Mol. Carcinog. 54, 249-260 (2015).

48. Chakraborti, C. K. New-found link between microbiota and obesity. World J. Gastrointest. Pathophysiol. 6, 110-119 (2015).

49. Duncan, S. H. et al. Human colonic microbiota associated with diet, obesity and weight loss. Int. J. Obes. (Lond.) 32, 1720-1724 (2008).

50. Harris, K., Kassis, A., Major, G. \& Chou, C. J. Is the gut microbiota a new factor contributing to obesity and its metabolic disorders? J. Obes. 2012, 879151 (2012).

51. Silva, L. G., Ferguson, B. S., Avila, A. S. \& Faciola, A. P. NaP and NaB effects on HDAC activity, histone acetylation, and inflammatory gene expression in bovine mammary epithelial cells. J. Anim. Sci. 96, 5244-5252 (2018).

52. Ma, N., Abaker, J. A., Bilal, M. S., Dai, H. \& Shen, X. NaB improves antioxidant stability in sub-acute ruminal acidosis in dairy goats. BMC Vet. Res. 14, 275 (2018).

53. Mathew, O. P., Ranganna, K. \& Milton, S. G. Involvement of the antioxidant effect and anti-inflammatory response in butyrate-inhibited vascular smooth muscle cell proliferation. Pharmaceuticals (Basel) 7, 1008-1027 (2014).

54. Zhang, Q. G. et al. Critical role of NOX in neuronal oxidative damage and microglia activation following traumatic brain injury. PLOS ONE 7, e34504 (2012).

55. Fakhruddin, S., Alanazi, W. \& Jackson, K. E. Diabetes-induced reactive oxygen species: mechanism of their generation and role in renal injury. J. Diabetes Res. 2017, 8379327 (2017).

56. Oh, J. Y. et al. 17ß-Estradiol protects mesenchymal stem cells against high glucose-induced mitochondrial oxidants production via NRF2/Sirt3/MnSOD signaling. Free Radic. Biol. Med. 130, 328-342 (2019).

57. Crispi, S. The dual role played by p21 may influence the apoptotic or antiapoptotic fate in cancer. J. Cancer Res. Updates 1, https://doi.org/10.6000/19292279.2012.01.02.5 (2012).

58. Bourassa, M. W., Alim, I., Bultman, S. J. \& Ratan, R. R. Butyrate, neuroepigenetics and the gut microbiome: Can a high fiber diet improve brain health? Neurosci. Lett. 625, 56-63 (2016)

59. Ruemmele, F. M. et al. Butyrate induced Caco-2 cell apoptosis is mediated via the mitochondrial pathway. Gut 52, 94-100 (2003).
60. Ahmed, S. M., Luo, L., Namani, A., Wang, X. J. \& Tang, X. NRF2 signaling pathway: pivotal roles in inflammation. Biochim. Biophys. Acta Mol. Basis Dis. 1863, 585-597 (2017).

61. Liu, T., Zhang, L., Joo, D. \& Sun, S. C. NF-kB signaling in inflammation. Sig. Transduct. Target Ther 2, https://doi.org/10.1038/sigtrans.2017.23 (2017).

62. Wardyn, J. D., Ponsford, A. H. \& Sanderson, C. M. Dissecting molecular cross-talk between NRF2 and NF-KB response pathways. Biochem. Soc. Trans. 43, 621-626 (2015).

63. Greene, W. C. \& Chen, L. F. Regulation of NF-KB action by reversible acetylation. Novartis Found. Symp. 259, 208-217 (2004). discussion 218-225.

64. Hunter, T. \& Karin, M. The regulation of transcription by phosphorylation. Cell 70, 375-387 (1992).

65. Karin, M. \& Hunter, T. Transcriptional control by protein phosphorylation: signa transmission from the cell surface to the nucleus. Curr. Biol. 5, 747-757 (1995).

66. Park, J. M., Jo, S. H., Kim, M. Y. Kim, T. H. \& Ahn, Y. H. Role of transcription factor acetylation in the regulation of metabolic homeostasis. Protein Cell 6, 804-813 (2015).

67. Thiagarajan, D., Vedantham, S., Ananthakrishnan, R., Schmidt, A. M. \& Ramasamy, R. Mechanisms of transcription factor acetylation and consequences in hearts. Biochim. Biophys. Acta 1862, 2221-2231 (2016).

68. Zhao, L. J., Subramanian, T., Zhou, Y. \& Chinnadurai, G. Acetylation by p300 regulates nuclear localization and function of the transcriptional corepressor CtBP2. J. Biol. Chem. 281, 4183-4189 (2006).

69. Klindworth, $A$. et al. Evaluation of general 165 ribosomal RNA gene PCR primers for classical and next-generation sequencing-based diversity studies. Nucleic Acids Res. 41, el (2013).

70. Davis, M. P., van Dongen, S., Abreu-Goodger, C., Bartonicek, N. \& Enright, A. J. Kraken: a set of tools for quality control and analysis of high-throughput sequence data. Methods 63, 41-49 (2013).

71. Bolyen, E. et al. Reproducible, interactive, scalable and extensible microbiome data science using QIIME 2. Nat. Biotechnol. 37, 852-857 (2019).

72. Martin, M. CUTADAPT removes adapter sequences from high-throughput sequencing reads. EMBnet.journal 17, https://doi.org/10.14806/ej.17.1.200 (2011).

73. Callahan, B. J. et al. DADA2: high-resolution sample inference from Illumina amplicon data. Nat. Methods 13, 581-583 (2016).

74. DeSantis, T. Z. et al. Greengenes, a chimera-checked $16 \mathrm{~S}$ rRNA gene database and workbench compatible with ARB. Appl. Environ. Microbiol. 72, 5069-5072 (2006).

75. Parks, D. H., Tyson, G. W., Hugenholtz, P. \& Beiko, R. G. STAMP: statistical analysis of taxonomic and functional profiles. Bioinformatics 30, 3123-3124 (2014).

76. Skoglund, J. Quantification of SCFAs in serum and plasma. Unpublished bachelor's thesis, http://urn.kb.se/resolve?urn=urn:nbn:se:slu:epsilon-s-6102, Swedish University of Agricultural Sciences, Sweden (2016).

77. Gustafsson, N. et al. Fast live-cell conventional fluorophore nanoscopy with ImageJ through super-resolution radial fluctuations. Nat. Commun. 7, 12471 (2016). 